\title{
Reconstruction of the 3D pressure field and energy dissipation of a Taylor droplet from a $\mu$ PIV measurement
}

\author{
Ulrich Mießner $^{1}$ (D) $\cdot$ Thorben Helmers $^{1} \cdot$ Ralph Lindken $^{2} \cdot$ Jerry Westerweel $^{3}$
}

Received: 7 October 2020 / Revised: 15 March 2021 / Accepted: 16 March 2021 / Published online: 31 March 2021

(c) The Author(s) 2021

\begin{abstract}
In this study, we reconstruct the 3D pressure field and derive the 3D contributions of the energy dissipation from a 3D3C velocity field measurement of Taylor droplets moving in a horizontal microchannel $\left(\mathrm{Ca}_{c}=0.0050, \operatorname{Re}_{c}=0.0519\right.$, Bo $=0.0043, \lambda=\frac{\eta_{d}}{\eta_{c}}=2.625$ ). We divide the pressure field in a wall-proximate part and a core-flow to describe the phenomenology. At the wall, the pressure decreases expectedly in downstream direction. In contrast, we find a reversed pressure gradient in the core of the flow that drives the bypass flow of continuous phase through the corners (gutters) and causes the Taylor droplet's relative velocity between the faster droplet flow and the slower mean flow. Based on the pressure field, we quantify the driving pressure gradient of the bypass flow and verify a simple estimation method: the geometry of the gutter entrances delivers a Laplace pressure difference. As a direct measure for the viscous dissipation, we calculate the 3D distribution of work done on the flow elements, that is necessary to maintain the stationarity of the Taylor flow. The spatial integration of this distribution provides the overall dissipated energy and allows to identify and quantify different contributions from the individual fluid phases, from the wall-proximate layer and from the flow redirection due to presence of the droplet interface. For the first time, we provide deep insight into the 3D pressure field and the distribution of the energy dissipation in the Taylor flow based on experimentally acquired 3D3C velocity data. We provide the 3D pressure field of and the 3D distribution of work as supplementary material to enable a benchmark for CFD and numerical simulations.
\end{abstract}

Ulrich Mießner

miessner@uvt.uni-bremen.de

Thorben Helmers

helmers@uvt.uni-bremen.de

Ralph Lindken

ralph.lindken@hs-bochum.de

Jerry Westerweel

J.Westerweel@tudelft.nl

1 Department of Environmental Process Engineering (UVT), University of Bremen, Leobener Str. 6, 28359 Bremen, Germany

2 Thermo- and Fluiddynamics, Bochum University of Applied Sciences, LennershofstraSSe 140, 44801 Bochum, Germany

3 Laboratory of Aero- and Hydrodynamics (AHD), Delft University of Technology, Mekelweg 2, 2628 CD Delft, Netherlands 


\section{Graphical abstract}

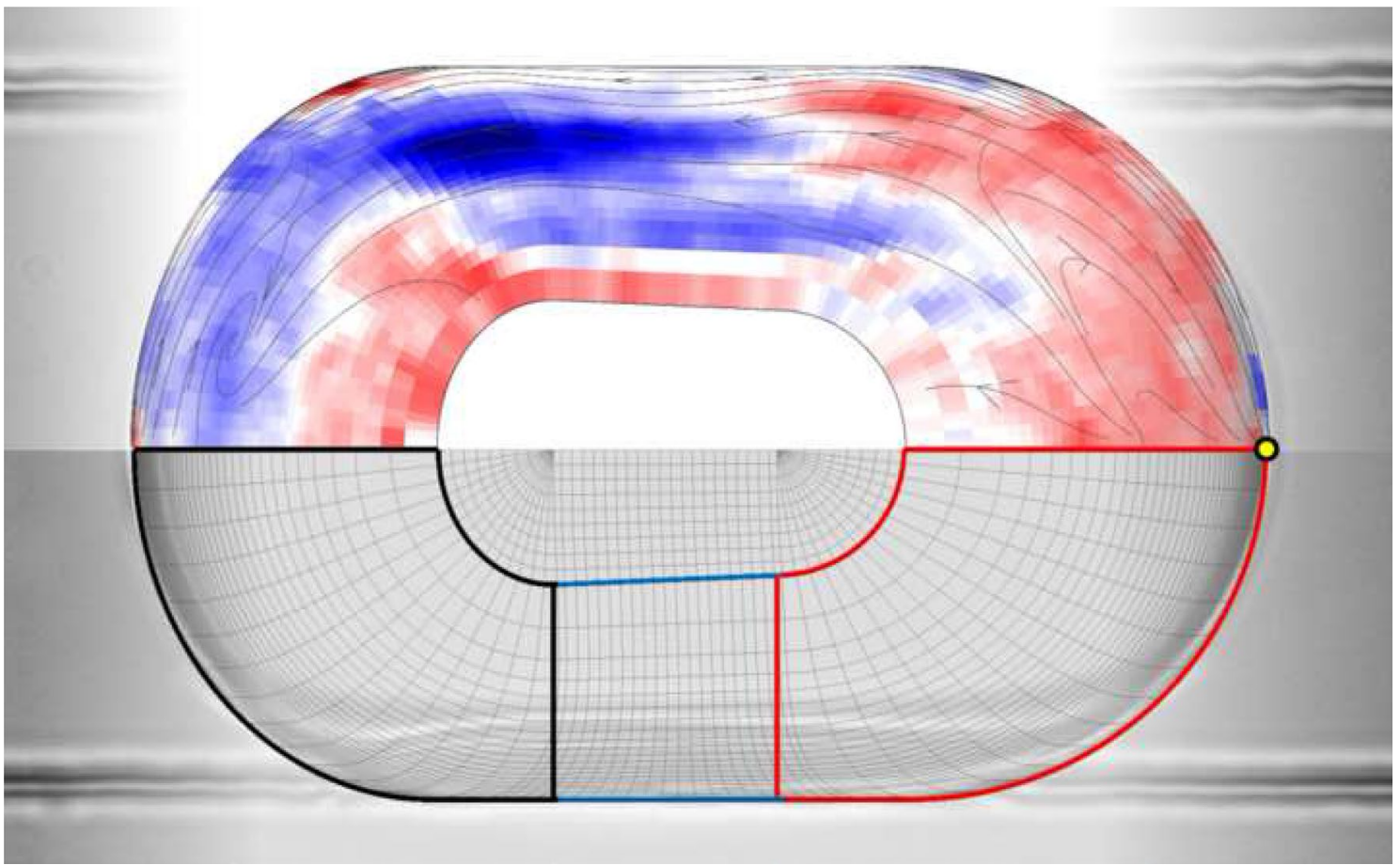

\section{List of symbols}

\section{Acronyms}

2D

$3 \mathrm{C}$

3D

$\mu \mathrm{PIV}$

CCD

CFD

const

DRIE

FF

Nd:YAG Neodymium-doped yttrium aluminum garnet crystal

PDMS Polydimethylsiloxane

RI Refractive index

\section{Dimensionless quantities}

$\lambda \quad$ Viscosity ratio

$\mathcal{K} \quad$ Density ratio

$a_{r} \quad$ Aspect ratio

Bo Bond number

$\mathrm{Ca} \quad$ Capillary number

Mo Morton number

Oh Ohnesorge number
Re Reynolds number

We Weber number

\section{Greek symbols}

$\delta \quad$ Thickness

$\eta \quad$ Dynamic viscosity

$\Gamma \quad$ Droplet interface

$\kappa \quad$ Curvature

$\Phi \quad \Phi$-direction

$\rho \quad$ Density

$\sigma \quad$ Interfacial tension

$\tau \quad$ Shear

$\varepsilon \quad$ Phase fraction

\section{Roman symbols}

$\tilde{y} \quad$ Dimesnionless wall distance

A Area

$c \quad$ Concentration

$F \quad$ Force

$H \quad$ Height

$L \quad$ Length

$P \quad$ Power

$p \quad$ Pressure

$Q \quad$ Volume flow rate

$R \quad$ Radius

$r \quad$ Radial direction 


$\begin{array}{ll}s & \text { Distance } \\ t & \text { Time } \\ U & \text { Velocity } \\ u & \text { Velocity } \\ V & \text { Volume } \\ V_{\mathrm{FF}} & \text { Volume of the FF-junction } \\ W & \text { Width } \\ x & \text { X-Direction } \\ y & \text { y-Direction } \\ z & \text { Z-Direction } \\ \mathrm{N} & \text { Number of points }\end{array}$

\section{Superscripts}

$\begin{array}{ll}j & \text { Index } \\ k & \text { Index } \\ * & \text { Normalized } \\ \text { dyn } & \text { Dynamic } \\ \text { stat } & \text { Static }\end{array}$

\section{Subscripts}

$\begin{array}{ll}c & \text { Continuous phase } \\ d & \text { Disperse phase } \\ g & \text { Gutter } \\ i & \text { Index } \\ o & \text { Orifice } \\ \text { tot } & \text { Total } \\ \text { ch } & \text { Channel } \\ \text { core } & \text { Core of the flow } \\ \text { dyn } & \text { Dynamic } \\ \text { HP } & \text { Hagen-Poiseuille } \\ \text { LP } & \text { Laplace } \\ \text { ref } & \text { Reference } \\ \text { res } & \text { Resulting } \\ \text { stat } & \text { Static } \\ \text { wall } & \text { Wall of the microchannel } \\ \text { Other symbols } \\ \mathcal{W}\end{array}$

\section{Introduction}

The applications of microscopic two-phase flow involve medical (Piao et al. 2015), biological (Wolf et al. 2015), chemical (Lang et al. 2012) and thermal processes (Leung et al. 2010). Chou et al. (2015) reviewed the application of Taylor flows in various fields.

Downscaled multiphase flows like Taylor flows are often realized in horizontal rectangular microchannel structures. They offer a variety of advantages for process engineering purposes: The increased specific surface area of the flow enhances heat and mass transfer (Bandara et al. 2015) and allows precise handling of sample volumes (Garstecki et al. 2006; Whitesides 2006). Seemann et al.
(2011) propose the application of Taylor droplets to enable high-speed processing without cross-contamination. The continuous phase slugs separate individual droplets, and a thin lubricating wall-film prevents the contact between the wall and the disperse phase. Besides the lubricating wallfilms, Taylor flows in rectangular microchannels feature a bypass flow through the continuous phase-filled corners (Kreutzer et al. 2005), the so-called gutters (van Steijn et al. 2009).

To control the flow stability of subsequent Taylor droplets, one needs to understand and control the pressure drop inside and outside the disperse phase. The challenge becomes obvious when considering microchannel-parallelization as a commonly used strategy to increase the throughput. Uniform flow conditions are desirable to achieve continuous and stable processes in all branches of a parallelized microreactor (Antweiler et al. 2016). Fluctuations of the two-phase flow change the product quality, since the process deviates from the optimal working point.

The stabilization of parallel flows requires to control the individual droplet formation at low capillary number and low Reynolds number. This formation process feedbacks sensitively to downstream pressure changes in a microchannel. The droplet shedding frequency and the droplet length are affected by pressure shifts (Ward et al. 2005). At a constant volume flow rate and for a given surfactantfree material combination between two immiscible phases, only the droplet length determines its hydraulic resistance. The hydraulic resistance influences the actual droplet velocity, which finally determines the droplet residence time in a microchannel (Helmers et al. 2019b). Thus, to control the flow stability of a Taylor droplet chain one needs to understand and control the pressure drop inside and outside the disperse phase.

Droplets in rectangular capillaries are less often addressed theoretically and fewer models exist than for Taylor flow in circular capillaries - pioneering work for the latter has been published by Bretherton (1961). An early correlation for rectangular capillaries that describes the pressure drop was published by Lockhart and Martinelli (1949) in the form of friction factors. To date, a variety of analytical models have been published: The relevant forces have been explained by Kreutzer et al. (2005b) and later expanded by Abiev (2011). The pressure profile is analyzed, and the relevant flows for the pressure drop are recognized (Abiev 2017). This author suggests to use the shape of the Taylor droplet interface to determine the local pressure distribution. Balestra et al. (2018) and Vivekanand and Raju (2019) add simulative work to the problem. Yue et al. (2014) developed a correlation for liquid/liquid slug flow in rectangular microchannels, and Ładosz and von Rohr (2018) extended their models and focused on the influence of the wall-film as proposed by Jovanović et al. (2011). 
Experimentally, a variety of measurement approaches have been used to characterize the pressure fluctuations and frictional pressure drop caused by Taylor droplets in microchannels. Techniques such as optical tweezers (Jin et al. 2012), Laplace-pressure sensors (Abate et al. 2012; Romero and Abate 2012; Xu et al. 2014) and also membrane cavities in DRIE-etched reactors have been used (Molla et al. 2011) in devices of small channel height $H_{\mathrm{ch}}<500 \mu \mathrm{m}$. In larger microchannels, mostly adapted conventional pressure sensors were applied, which allow to measure the total pressure at the entrance and exit of the microchannel to calculate the pressure drop along the entire channel (Wang et al. 2015; Mac Giolla Eain et al. 2015; Nayak et al. 2018). Despite relying on integral information about entire droplet chains, Wang et al. (2014) showed the importance of the wall contact angles for the magnitude of the pressure drop. The pressure drop caused by droplet trains in pressure-driven flows has been examined by Jakiela (2016), who also worked on the influence of droplet length and presents a measurement device for networks.

All of these approaches measure either the pressure at a distinct position at the channel walls or integrally over a number of droplets. For modeling purposes and further understanding of the underlying effects, spatially resolved pressure measurements are necessary. However, a 3D pressure field of a Taylor droplet extracted from a measurement has not yet been published.

With the intention to close this gap, we base this study on the $\mu$ PIV-measurement of Mießner et al. (2020). Their study provides the 3D3C velocity field in and around quasi-stationary Taylor droplets moving in a horizontal square microchannel. In the underlying experiment, the surface tension forces dominate the viscous forces. Thus, the capillary number is small $\mathrm{C} a_{\mathrm{c}}=\frac{\bar{U} \eta_{\mathrm{c}}}{\sigma} \ll 1$. Herein, $\sigma$ is the interface tension, $\eta_{\mathrm{c}}$ denotes the dynamic viscosity of the continuous phase and $\bar{U}=\frac{Q_{\mathrm{c}}+Q_{\mathrm{d}}}{A_{\mathrm{ch}}}$ represents the total superficial velocity of the flow. This average velocity is derived from the total volume flow through the area of the channel cross-section $A_{\text {ch }}$, where $Q_{\mathrm{c}}$ and $Q_{\mathrm{d}}$ are the volume flows of the continuous and the disperse phase, respectively.

In this context, the droplet velocity $u_{\mathrm{d}}$ is not useful for the definition of dimensionless numbers, since it is a flow dependent variable (Helmers et al. 2019b): Continuous phase bypasses the droplet through the gutters from the droplet front to its back and causes a relative velocity $u_{\text {rel }}=u_{\mathrm{d}}-\bar{U}$ of the Taylor droplet. Therefore, the droplet travels faster than the average flow $\bar{U}$ depending among other variables on the magnitude of the average flow velocity. Please note that the total volume flow calculated from the velocity field (Mießner et al. 2020) is smaller (94.44\% \pm 1.2 $\%$ ) than the superimposed volume flow $Q_{\text {tot }}$ discussed above due to channel deformation. This circumstance impacts the direct calculation of the relative velocity from the setup data given in Table 1.

A low Reynolds number $\mathrm{R} e_{\mathrm{c}}=\frac{\rho_{\mathrm{c}} \bar{U} H_{\mathrm{ch}}}{\eta_{\mathrm{c}}} \leq 1$ relates the weak inertia forces to the dominating viscous forces and indicates Stokes-flow conditions. Herein, $\rho_{\mathrm{c}}$ denotes the density of the continuous phase, and $H_{\mathrm{ch}}$ is the channel height. A low Bond number $\mathrm{B} o=\frac{\Delta \rho g H_{\mathrm{ch}}^{2}}{\sigma} \ll 1$ indicates a negligible influence of the buoyancy forces on the droplet shape. Herein $\Delta \rho$ denotes the density difference between the two phases, and $g$ is the gravitational acceleration. The governing forces for the investigated Taylor flow given in descending order are the interfacial tension forces, the viscous forces, the buoyancy forces and the inertia forces $\left(\mathrm{Ca}_{c}=0.0050, \mathrm{Re}_{c}=0.0519\right.$, Bo $\left.=0.0043\right)$.

In the present study, we use the measured 3D3C velocity field of Taylor droplets (Mießner et al. 2020) twofold: to reconstruct and investigate the $3 \mathrm{D}$ pressure field as well as to derive and discuss the contributions of the energy dissipation in the Taylor flow.

- At first, we apply the Navier-Stokes equation to the velocity field of the Taylor flow and the subsequent spatial integration calculates the 3D field of the pressure in and around a Taylor droplet. In this context, the calculation of the momentum thickness helps to discriminate between wall-influenced flow and the remaining core of the flow. While the gradient of the pressure inside the wall-layer points downstream as expected, we experimentally show a pressure gradient inversion with respect to the flow direction, and provide experimental evidence of the postulation of Abiev (2017). This reversed pressure gradient in the core of the flow drives the bypass flow of continuous phase through the corners (gutters) and gives rise to the Taylor droplet's relative velocity (Helmers et al. 2019b). To quantify the driving pressure gradient along the gutter, the Taylor droplet interface approximation of Mießner et al. (2019) is applied twice: to evaluate the experimental data of the pressure field directly at the interface position and subsequently to establish and verify an estimation method for the calculation of the gutter gradient.

- Secondly, we use the viscous forces of the flow field and the velocity distribution to calculate the $3 \mathrm{D}$ field of work done on the flow elements. This work maintains the stationarity of the Taylor flow. The spatial integration of this distribution allows to identify and quantify different contributions to the losses of the flow: e.g. the properties of the individual fluid phases, the wall-influence and the flow redirection by the interface (Fig. 1). The total work done on the flow represents the mechanical energy introduced to the Taylor flow to maintain stationarity, i.e., to compensate for the viscous dissipation. 
Passage of a single Taylor droplet

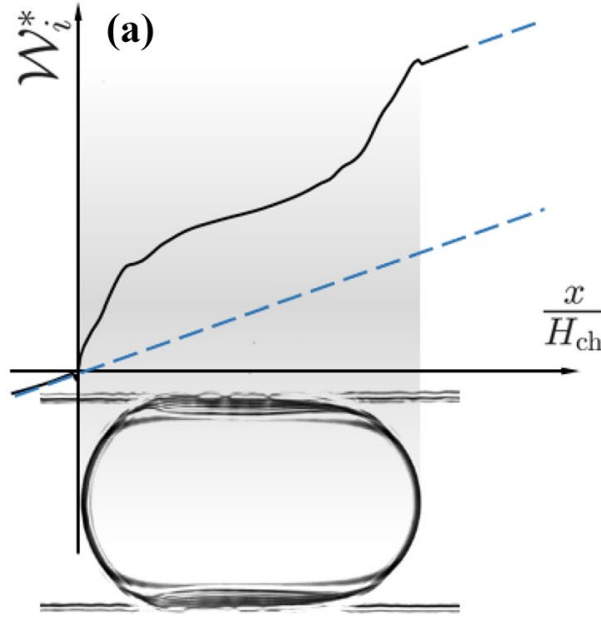

Fig. 1 Qualitative sketch comparing the viscous dissipation of a single-phase flow with that of a Taylor flow: In a stationary flow subjected to internal friction, energy is dissipated and work $\mathcal{W}_{i}^{*}$ needs to be done on the flow to compensate for the loss of mechanical energy. a A stationary laminar viscous single-phase flow in a straight microchannel (blue dashed line) needs work done on the flow to solely overcome the wall-shear, and thus the increase of work inflow direc-

\section{Passage of multiple Taylor droplets}

(b)

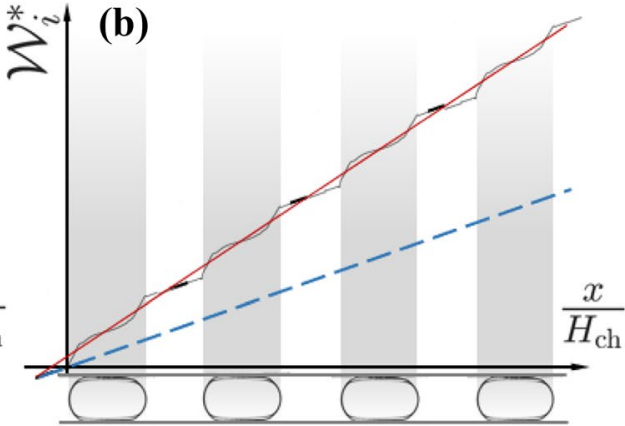

- - - Single phase Poiseuille flow

$\begin{aligned} \text { _ } & \text { Taylor flow } \\ & \text { with increased slope } \\ => & \text { increased pressure drop }\end{aligned}$

tion is linear. The presence of a droplet interface redirects the flow field, adds energy loss to the shear contribution and increases the amount of work done on the flow at the droplet caps, where the directional change takes place (solid black line). b In a stationary Taylor droplet chain, the contribution of each droplet sums up to an overall increased amount of work done on the flow (thin red line) in comparison with the single-phase flow
Table 1 Flow conditions of the measured Taylor flow (Mießner et al. 2020). Geometric parameters, fluidic and material parameters, and the governing dimensionless numbers are displayed

\begin{tabular}{lllllllll}
\hline Dimensionless numbers & & \multicolumn{2}{l}{ Geometric parameters } & & \multicolumn{2}{l}{ Fluidic/material parameters } \\
\hline $\mathrm{Ca}_{c}$ & 0.0050 & - & $W_{\mathrm{ch}}$ & 104 & $\mu$ & $Q_{\mathrm{d}}$ & 1.5 & $\mu \mathrm{min}^{-1}$ \\
$\mathrm{Re}_{c}$ & 0.0511 & - & $H_{\mathrm{ch}}$ & 96 & $\mu$ & $Q_{\mathrm{c}}$ & 1.5 & $\mu \mathrm{min}^{-1}$ \\
$\mathrm{Oh}$ & 0.3107 & - & $A_{\mathrm{ch}}$ & 9984 & $\mu^{2}$ & $Q_{\mathrm{tot}}$ & 3.0 & $\mu \mathrm{min}^{-1}$ \\
$\mathrm{Bo}$ & 0.0043 & - & $V_{\mathrm{FF}}$ & 1038336 & $\mu^{3}$ & $\bar{U}$ & 5.0 & $\mathrm{~mm} \mathrm{~s}^{-1}$ \\
$\mathrm{We}$ & 0.0003 & - & $a_{\mathrm{r}}$ & 1.08 & - & $u_{\mathrm{d}}$ & 4.7 & $\mathrm{~mm} \mathrm{~s}^{-1}$ \\
$\mathrm{Mo}$ & 0.0004 & - & $L_{\mathrm{d}} / W_{\mathrm{ch}}$ & 1.58 & - & $\eta_{\mathrm{d}}$ & 0.021 & $\mathrm{~Pa} \mathrm{~s}$ \\
$\alpha$ & 1.0 & - & $L_{\mathrm{g}} / W_{\mathrm{ch}}$ & 0.34 & - & $\eta_{\mathrm{c}}$ & 0.008 & $\mathrm{~Pa} \mathrm{~s}$ \\
$\varepsilon_{\mathrm{d}}$ & 0.5 & - & $L_{\mathrm{ch}} / W_{\mathrm{ch}}$ & 500 & - & $\rho_{\mathrm{d}}$ & 1186 & $\mathrm{Kg} \mathrm{m}^{-3}$ \\
$\lambda=\eta_{\mathrm{d}} / \eta_{\mathrm{c}}$ & 2.625 & - & $W_{\mathrm{o}} / W_{\mathrm{ch}}$ & 0.5 & - & $\rho_{\mathrm{c}}$ & 830 & $\mathrm{Kg} \mathrm{m}^{-3}$ \\
$\mathcal{K}=\rho_{\mathrm{d}} / \rho_{\mathrm{c}}$ & 1.427 & - & $L_{\mathrm{o}} / W_{\mathrm{ch}}$ & 0.5 & - & $\sigma$ & 0.008 & $\mathrm{~N} \mathrm{~m}^{-1}$ \\
\hline
\end{tabular}

The denomination is given in the list of symbols
Three major benefits can be drawn from this work: (i) the experimental validation of the analytical quantification method that estimates the magnitude of the reversed pressure gradient in the gutter of Taylor droplets, (ii) the 3D pressure field and (iii) the 3D distribution of mechanical work done on the flow. The pressure and the data of work done are offered as supplementary material to enable a benchmark for CFD and numerical simulations (supplementaryDataPressureTaylorDroplet.mat).

\section{Materials and methods}

At first, we give a short overview on the experimental conditions and methods applied to acquire the 3D3C velocity field of a moving Taylor droplet. Secondly, a force balance at the interface relates the pressure field of the flow to the Laplace-pressure distribution on the interface. Then, we apply the Navier-Stokes equation with the measured 
velocity data to calculate the pressure distribution inside and outside the Taylor droplet. Next, the 3D scalar field of the work done on the flow is derived from the velocity field, and the interface approximation (Mießner et al. 2019) is applied to deduce the Laplace-pressure distribution of the motion-deformed interface. Subsequently, three different methods are explained to quantify the overall energy loss of the flow inside and outside the Taylor droplet. Finally, we calculate the momentum thickness as a geometric measure to discriminate between a wallproximate flow layer and the core of the flow.

\subsection{Experimental methods}

This study processes the results of the experimental work of Mießner et al. (2020). In order to deliver experimental context, we very briefly provide some specifications of the experimental setup; an in-depth description of the experimental details of the $\mu$ PIV-study is given by Mießner et al. (2019) and Mießner et al. (2020).

Steady and pulsation-free volumetric flow rates of the disperse phase $Q_{\mathrm{d}}$ (water-glycerin) and the continuous phase $Q_{\text {c }}$ (octanol) establish a regular Taylor droplet train behind the pin-hole of a flow-focussing (FF) device. The Taylor flow's volume fraction is $\varepsilon_{\mathrm{d}}=0.5$. A thin octanol film $(\delta \lesssim$ $1 \mu \mathrm{m})$ always separates the droplets from the smooth hydrophobic wall material (polydimethylsiloxane, abbreviated as PDMS). The refractive index (RI) of the droplet phase is matched to that of the continuous phase flow. The flow profile of the Taylor droplets is optically measured $5 \mathrm{~mm}$ downstream of the FF-junction using $\mu$ PIV. The side walls of the channel are not ideally parallel and enclose an angle of $4.7^{\circ}$. We neglect this small trapezoidal deviation and consider the microchannel cross section to be rectangular $\left(W_{\mathrm{ch}}\right.$ $\left.=104 \mu \mathrm{m}, H_{\mathrm{ch}}=96 \mu \mathrm{m}\right)$. Both phases are seeded with fluorescent tracer particles (Rhodamine B coating) of different seeding density $\left(c_{d} \approx 0.7 c_{c}\right)$ to enable optical discrimination between the phases. A pulsed Nd:YAG laser excites the fluorescence of the particles. Images of the fluorescent tracer signal are recorded with a CCD-camera through a microscope. The z-position of the focal plane is controlled with a piezo-stepper (MIPOS500SG, Piezosystem Jena $\mathrm{GmbH}$ with a precision of $8 \mathrm{~nm}$ ), which enables to scan through the measurement volume. Per measurement plane a minimal number of 120 valid images is necessary for the $\mu \mathrm{PIV}$ evaluation. The symmetry of the microchannel allows to reduce the amount of data to one half of the measurement volume. After image preprocessing, plane-wise ensemblePIV evaluations results in a 3D2C velocity field of a mean quasi-stationary Taylor droplet in the measurement domain. The out-of-plane velocity is reconstructed based on the conservation of mass. A detailed description of the method is given by Brücker $(1995,1997)$. An overview of the flow conditions is given in Table 1 .

\subsection{Force balance at the interface}

The interface shape of a Taylor droplet changes from static conditions (without flow) to dynamic conditions (Mießner et al. 2019; Helmers et al. 2019b). At steady state, a clean droplet interface $\mathrm{d} A$ between two immiscible phases is subject to the following balance of normal forces (Fig. 2).

$-F_{\mathrm{LP}, \mathrm{c}}^{j}+F_{\mathrm{p}, \mathrm{c}}^{j}+F_{u, \mathrm{c}}^{j}+F_{\mathrm{LP}, \mathrm{d}}^{j}-F_{\mathrm{p}, \mathrm{d}}^{j}-F_{u, \mathrm{~d}}^{j}=0$.

Herein, $F_{u, i}^{j}$ refers to the normal forces induced by the adjoining flow conditions, $F_{p, i}^{j}$ indicates the normal forces exerted by the pressure and $F_{\mathrm{LP}, \mathrm{i}}^{j}$ represents the normal forces that arise from the energetic molecular interaction inside the individual phases. The latter macroscopically results in the surface tension forces and is related to the Laplace-pressure. The index $j$ refers to the flow conditions with $j=$ stat denoting the static case (without flow) and $j=$ dyn representing the influence of velocity field. The index $i$ refers to either the disperse phase $i=\mathrm{d}$ or the continuous phase $i=\mathrm{c}$. Tangential forces immediately induce flow on ideal clean interfaces and do not contribute to this balance. The experimental results of Mießner et al. (2020) clearly show interface mobility and support the assumption of a minor interface contamination and an ideal interface behavior.

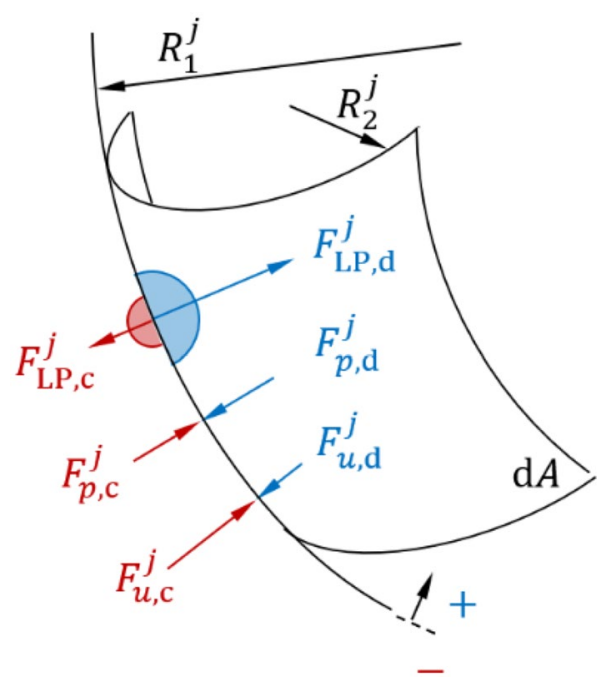

Fig. 2 Forces $F_{k}^{j}$ at a curved interface $\mathrm{d} A$ between two immiscible phases ( $\mathrm{d}$-disperse phase, $\mathrm{c}$-continuous phase) in combination with the principle radii $R_{1,2}^{j}$ to link the Laplace-pressure to the adjoining flow field. The superscript $j$ refers to static $(j=$ stat.) versus dynamic $(j=$ dyn.) flow conditions. The positive reference direction points into the disperse phase 
Equation 1 allows to compare the force balance at static conditions ( $j=$ stat) with the force balance at dynamic conditions $(j=$ dyn). A consideration of the individual terms simplifies the equation. The forces exerted by the pressure on the respective sides of the interface cancel out $\left(F_{p, \mathrm{~d}}^{j}=F_{p, \mathrm{c}}^{j}\right)$. Without flow, the velocity forces of the static conditions vanish $\left(F_{u, i}^{\text {stat }}=0\right)$. We combine the forces across the interface to a resulting force that can be calculated from the Laplace-pressure $\Delta F_{\mathrm{LP}}^{j}=+F_{\mathrm{LP}, \mathrm{d}}^{j}-F_{\text {LP,c }}^{j}$. A separation between the flow-induced forces and the interface tension forces links the shape deformation directly to the adjoining flow field:

$\Delta F_{\mathrm{LP}}^{\mathrm{stat}}-\Delta F_{\mathrm{LP}}^{\mathrm{dyn}}=+F_{u, \mathrm{c}}^{\mathrm{dyn}}-F_{u, \mathrm{~d}}^{\mathrm{dyn}}$

A division of the forces by the interface area $\mathrm{d} A$ results in a relation between the flow-induced pressure field and the Laplace-pressure difference between the static and the dynamic interface shape:

$$
\begin{aligned}
\left(\Delta p_{\mathrm{LP}}^{\mathrm{stat}}-\Delta p_{\mathrm{LP}}^{\mathrm{dyn}}\right) & =+p_{u, \mathrm{c}}^{\mathrm{dyn}}-p_{u, \mathrm{~d}}^{\mathrm{dyn}} \\
\Delta p_{\mathrm{LP}}^{\text {(stat } \mapsto \mathrm{dyn})} & =+p_{u, \mathrm{c}}^{\mathrm{dyn}}-p_{u, \mathrm{~d}}^{\mathrm{dyn}}
\end{aligned}
$$

In disregard of the involved material parameters of the Taylor flow $\left(\sigma, \rho_{\mathrm{d}}, \rho_{\mathrm{c}}, \eta_{\mathrm{d}}, \eta_{\mathrm{c}}\right)$, the left-hand side of Eq. 3 consists of geometry information, while the right-hand side is based on the flow field. This allows a direct quantitative comparison between the curvature-dependent Laplace pressure of the interface geometry and the velocity field-dependent pressure difference at the interface.

\subsection{D pressure distribution}

Two approaches are available to compute the pressure distribution from the $\mu$ PIV measurements. One is based on the solution of the Poisson equation and requires a set of (Neumann/Dirichlet) boundary conditions to derive the pressure field (Gurka et al. 1999; Koschatzky et al. 2011; De Kat and van Oudheusden 2012). The second approach involves the direct integration of the momentum equation by means of finite differences. The main problem with the latter method is related to the accumulation of noise and integration error, which is successively incorporated into the derived pressure field (Baur 1999; Liu and Katz 2006; Jaw et al. 2009; Tronchin et al. 2015). Recently, Cai et al. (2020) have proposed a variational formulation for the pressure-from-velocity problem in two dimensions.

Charonko et al. (2010) reported that line integral methods perform better for the internal flow, while the pressure Poisson equation is superior for the external flow. De Kat and van Oudheusden (2012) proposed guidelines for the temporal and spatial resolution of the PIV-data. While the acquisition frequency has no relevance in the investigated quasi-stationary Taylor flow, the interrogation window size is supposed to be 5 times smaller than the flow structures to properly resolve the pressure features. In the presented case, the interrogation window size is about 14 times smaller than the vortex features.

In contrast to the applications of the above stated papers, the $\mu \mathrm{PIV}$-study incorporated into this paper deals with Taylor droplets at low $\mathrm{Ca}_{c}$ and $\mathrm{Re}_{c}$, i.e. the surface tension forces dominate the viscous forces and inertia plays a subordinate role. Thus, we decided to apply a direct integration scheme to obtain the pressure, since we address an internal flow and need to consider the second velocity derivatives of the viscous dissipation.

The Navier-Stokes equation for stationary incompressible viscous flows with negligible influence of body forces reads as follows:

$\rho(\mathbf{u} \cdot \nabla) \mathbf{u}=-\nabla p+\eta \nabla^{2} \mathbf{u}$

Based on the measured velocity field, the velocity gradient tensor is calculated together with the second derivatives. For the estimate of the pressure from the flow field, we directly integrate the Navier-Stokes equation. Since we cannot provide a reference pressure for the flow field, we omit the integration constant $(c=0)$.

$$
\begin{aligned}
p_{u, i}^{\mathrm{dyn}}= & -\rho_{i} \int_{V}(\mathbf{u} \cdot \nabla) \mathbf{u} \mathrm{d} \mathbf{x} \\
& +\eta_{i} \int_{V}\left(\nabla^{2} \mathbf{u}\right) \quad \mathrm{d} \mathbf{x}
\end{aligned}
$$

The direct integration procedure is done separately for the convective and the dissipative velocity contributions. Each integration step with respect to a coordinate axis is performed twice: Along and against the axis-direction. The two respective results are averaged to reduce the influence of error accumulation (Charonko et al. 2010). The interface approximation of Mießner et al. (2019) serves as a logical discriminator to attribute the material properties to the according phases. The convective contributions are added to the viscous terms to receive the entire $3 \mathrm{D}$ pressure field.

\subsection{D field of the work done on the flow}

Macroscopically, work is added to the experimentally investigated flow by a syringe pump that builds up a pressure gradient to establish the flow (kinetic energy) in the microchannel. To maintain a flow, the overall work added to the flow system needs to compensate for directional changes, potential energy changes and frictional losses. In the considered case, the system is quasi-stationary, isothermal and incompressible, volume forces have no effect and Stokes-flow conditions apply for a Newtonian fluid 
$\left(\operatorname{Re}_{c}=0.052\right)$. Thus, the work added by the pump to the stationary flow solely compensates for the total friction losses in the system.

The work to drive a single average quasi-stationary Taylor droplet through a microchannel is calculated by volume integration of the work done on individual fluid elements $\mathcal{W}$. The work done on the fluid elements composes a scalar field. For an individual element, the work is calculated from the forces $\mathbf{F}$ acting along its path $\mathbf{s}$.

$\mathcal{W}=\mathbf{F} \cdot \mathbf{s}$

The forces are derived from the total change of momentum I over time, with $m, \rho$ and $V$ being the mass, density and volume of the fluid element, respectively.

$\mathbf{F}=\frac{\mathrm{DI}}{\mathrm{D} t}=\frac{\mathrm{D} m \mathbf{u}}{\mathrm{D} t}=V \rho \frac{\mathrm{Du}}{\mathrm{D} t}$

The above-stated flow conditions (stationary Stokes-flow, etc.) simplify the Navier-Stokes equation in (4) to

$\boldsymbol{\nabla} p=\eta \boldsymbol{\nabla}^{2} \mathbf{u}$.

Thus, the only forces that cause a pressure change in the considered Taylor flow are the friction forces. The work done on a fluid element to compensate the friction forces is expressed as

$\mathcal{W}=\mathbf{F} \cdot \mathbf{s}=\left(V \eta \nabla^{2} \mathbf{u}\right) \cdot(\mathbf{u} t)$.

Herein, the vector of the element's path $\mathbf{s}$ is estimated with its velocity and a short period of reference time $\mathbf{s}=\mathbf{u} t$. A non-dimensional representation of the work done per fluid element emerges after division by a reference work. As reference, we chose the work done on a laminar pressure-driven $\left(\Delta p_{\mathrm{HP}, \mathrm{c}}\right)$ single-phase flow (Hagen-Poiseuille flow - HP) of continuous phase $\left(Q=Q_{\mathrm{tot}}\right)$ through the same square cross section $A_{\text {ch }}$.

$\mathcal{W}_{\text {ref }}=\mathbf{F} \cdot \mathbf{s}=\left(\Delta p_{\mathrm{HP}, \mathrm{c}} \cdot A_{\mathrm{ch}}\right)\left(\frac{Q_{\mathrm{tot}}}{A_{\mathrm{ch}}} t\right)$

The scalar 3D field of dimensionless work $\mathcal{W}^{*}$ reads as follows:

$\mathcal{W}^{*}=\frac{\mathcal{W}}{\mathcal{W}_{\text {ref }}}=\frac{\left(V \cdot \eta \nabla^{2} \mathbf{u}\right) \cdot(\mathbf{u} t)}{\left(\Delta p_{\mathrm{HP}, \mathrm{c}} Q_{\mathrm{tot}}\right) t}$

Negative values of the work indicate deceleration of the flow, while positive values represent acceleration of fluid elements.

The duration of the reference time can be chosen arbitrarily, since the considered Taylor flow is quasi-stationary in the relative frame of reference. This leads to the conclusion, that the dimensionless work $\mathcal{W}^{*}$ calculated above is equal to the dimensionless power loss $P^{*}$ and the dimensionless pressure drop $\Delta p^{*}$.

$$
\begin{aligned}
P^{*} & =\frac{P}{P_{\mathrm{ref}}}=\frac{\left(V \cdot \eta \nabla^{2} \mathbf{u}\right) \cdot(\mathbf{u})}{\left(\Delta p_{\mathrm{HP}, \mathrm{c}} Q_{\mathrm{tot}}\right)} \\
\Delta p^{*} & =\frac{\Delta p}{\Delta p_{\mathrm{ref}}}=\frac{Q_{\mathrm{tot}}^{-1}\left(V \cdot \eta \boldsymbol{\nabla}^{2} \mathbf{u}\right) \cdot(\mathbf{u})}{\Delta p_{\mathrm{HP}, \mathrm{c}}} \\
\mathcal{W}^{*} & \cong P^{*} \cong \Delta p^{*}
\end{aligned}
$$

Despite the equivalence of the dimensionless quantities, we proceed the presentation of this study based on the above introduced concept of work that needs to be done on the flow to overcome the viscous losses in order to maintain the flow stationary.

\subsection{Pressure distribution on the droplet interface}

The energetic minimization of the adhesion and cohesion forces between the molecules along and across the clean interface $\mathrm{d} A$ of the contacted phases (Fig. 2) determine its curvature at static flow conditions. The Laplace-pressure describes the macroscopic effect that relates the interface tension with the curvature of $\mathrm{d} A$ to the pressure in the disperse phase.

$\Delta p_{\mathrm{LP}}^{j}=\sigma\left(\frac{1}{R_{1}^{j}}+\frac{1}{R_{2}^{j}}\right)$

When the flow field close to the interface exerts normal forces onto the interface $\mathrm{d} A$, the curvature is deformed from the static shape ( $j=$ stat) and the Laplace-pressure is altered ( $j=$ dyn).

The pressure difference between both geometric states equals the pressure contribution exerted by the flow (Eq. 3). Thus, there are two methods to derive the pressure distribution on a moving Taylor droplet: i) With knowledge of the geometry of the interface shape, we calculate the curvature distribution and determine the Laplace-pressure distribution. ii) The evaluation of the 3D velocity field of a moving Taylor droplet delivers the pressure difference across the interface at the location of the interface.

An approximation of the Taylor droplet interface (Mießner et al. 2019) provides primary and secondary geometry information. Primary information means e.g. location, volume, interface area, while secondary information refers to e.g. the curvature distribution. The static and the dynamic shape of the droplet deliver the respective curvature distributions to calculate the curvature difference distribution $\Delta \kappa$. For the latter step, we apply the Matlab script "Surfature" (Claxton 2006): 


$$
\begin{aligned}
\Delta p_{\mathrm{LP}}^{\text {(stat } \mapsto \mathrm{dyn})} & =\sigma\left(\frac{1}{R_{1}^{\text {stat }}}+\frac{1}{R_{2}^{\text {stat }}}\right)-\sigma\left(\frac{1}{R_{1}^{\text {dyn }}}+\frac{1}{R_{2}^{\text {dyn }}}\right) \\
& =\sigma\left(\frac{R_{1}^{\text {dyn }}-R_{1}^{\text {stat }}}{R_{1}^{\text {stat }} R_{1}^{\text {dyn }}}+\frac{R_{2}^{\text {dyn }}-R_{2}^{\text {stat }}}{R_{2}^{\text {stat }} R_{2}^{\text {dyn }}}\right) \\
& =\sigma\left(\Delta \kappa_{1}^{\text {(stat } \mapsto \text { dyn })}+\Delta \kappa_{2}^{\text {(stat } \mapsto \text { dyn) }}\right)
\end{aligned}
$$

The expression in Eq. 14 is a direct geometric measure for the pressure exerted onto the interface. This scalar quantity is projected onto the interface and easily compared to the pressure derived from the velocity field.

To receive the flow-related pressure on the moving Taylor droplet interface, we subtract the pressure of the disperse phase $p_{u, \mathrm{~d}}^{\mathrm{dyn}}$ from pressure of the continuous phase $p_{u, \mathrm{c}}^{\mathrm{dyn}}$ (Eq. 3). The equation is valid only at the position of the interface, because here the Laplace-pressure emerges from the material property changes. We use the interface approximation (Mießner et al. 2019) to determine the interface location of the moving droplet. The resulting pressure difference is a scalar quantity that is also projected onto the interface and compared to the curvature-based Laplacepressure distribution.

\subsection{Quantification of the overall energy dissipation}

We quantify the overall energy loss of the Taylor flow by determination of the work $\mathcal{W}_{i}^{*}$ that is necessary to keep the observed flow section stationary (Sec. 2.4). To obtain a dimensionless quantity, the work done to keep up the Taylor flow is divided by the work that is necessary to maintain a Hagen-Poiseuille flow of continuous phase with the same total volume flow through the same cross section. The experimental data set offers three different derivation possibilities for the overall energy loss of a moving Taylor droplet:

(i) The shear forces $F_{\tau, x}$ at the wall and the drag forces $F_{\Gamma, x}$ at the droplet interface along the droplet deliver the total resisting force. The 2D distribution of the pressure on the surface of a moving Taylor droplet is used to estimate the drag forces of the droplet interface. The interface-grid is re-sampled into cylindrical coordinates (Fig. 3a) in order to correspond to the $\mathrm{x}$-axis grid of the velocity data. The $\mathrm{x}$-axis points in downstream direction. The cumulated resistance forces in the flow direction are calculated for the gutter region and the film region separately. The pressure $\Delta p_{\Gamma}$ on a surface area element $\mathrm{d} A$ (Fig. 3b) results in a force normal $\mathbf{n}_{0}$ to the area element. Its projection in flow direction $\mathbf{e}_{x, 0}$ contributes to the drag of the droplet (Fig. 3c). A summation of all resistance forces $F_{i, x}$ along the $\phi$-direction of the flow delivers the drag force distribution at the drop-

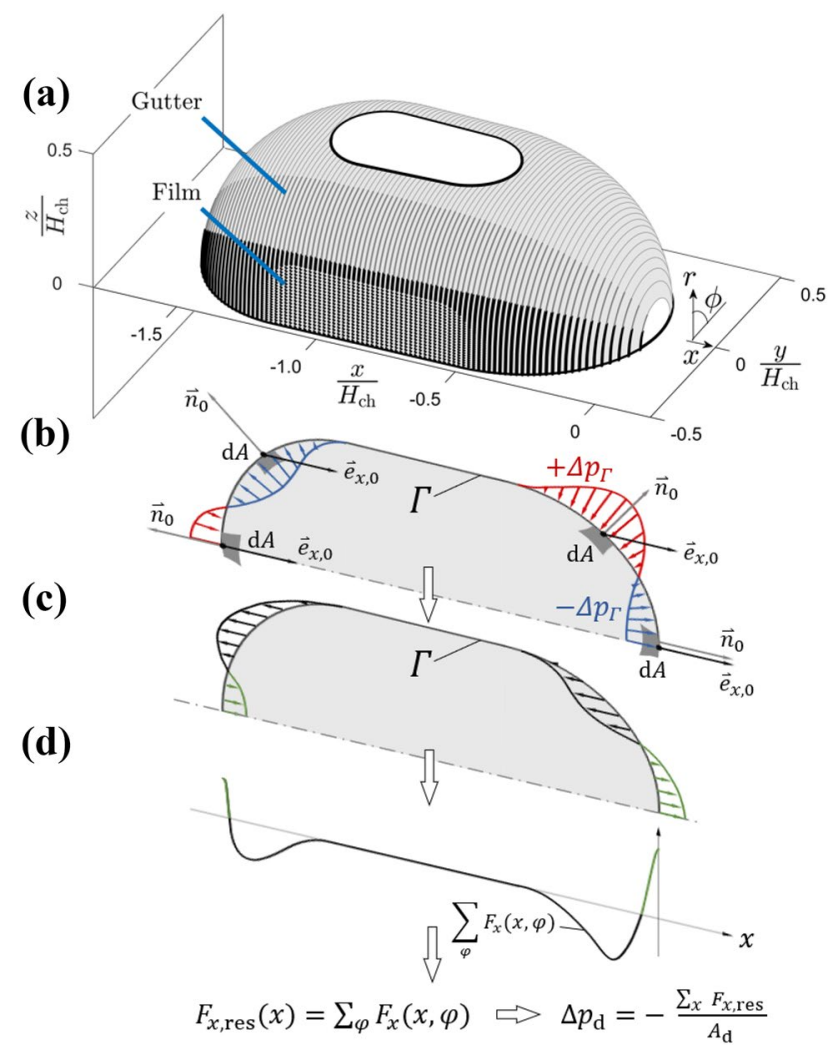

Fig. 3 Evaluation scheme of forces acting on the Taylor droplet interface. a Redefinition of the droplet interface grid into cylindrical coordinates to enable a calculation of the drag force, since the velocity data grid and the nodes of the interface do not coincide. The common $\mathrm{x}$-axis allows to compare the obtained profiles. The interface forces are evaluated in a gutter region and in a film region. The symmetry of the measurement domain is used to calculate the total drag force. b-c show the conversion scheme of the pressure at the interface $\Gamma$ of a moving Taylor droplet (b) into a drag force distribution in flow direction (c) to calculate the total drag force along the Taylor droplet length $L_{\mathrm{d}}(\mathbf{d})$

let interface $F_{x, \text { res }}$. A cumulative sum of the latter provides the drag force evolution along the Taylor droplet $\sum_{V} F_{\Gamma, x}$ (Fig. 3d).

$\mathcal{W}_{F}^{*}=\frac{\left(\sum_{V} F_{\tau, x}+\sum_{V} F_{\Gamma, x}\right) L_{\mathrm{d}}}{\left(\Delta p_{\mathrm{HP}, \mathrm{c}} A_{\mathrm{ch}}\right) L_{\mathrm{d}}}$

(ii) The pressure profile at the wall of the channel is an indicator for the pressure loss of the flow. The flow loses energy in the same rate as the profile changes. Thus, a linear fit of the inverted mean pressure profile at the wall serves as reference for the evolution of the pressure loss $\Delta \bar{p}_{\text {wall }}(x)$ along the droplet in flow direction. A measure for the drag forces is established after multiplication with the area of the channel cross section. The work done on the flow is received from the forces acting along the droplet path of length $L_{\mathrm{d}}$. 
$\mathcal{W}_{\Delta p, \text { wall }}^{*}=\frac{\left(\Delta \bar{p}_{\text {wall }}(x) A_{\mathrm{ch}}\right) L_{\mathrm{d}}}{\left(\Delta p_{\mathrm{HP}, \mathrm{c}} A_{\mathrm{ch}}\right) L_{\mathrm{d}}}$

(iii) The work distribution in the viscous flow field inside and outside the droplet is derived in Sec. 2.4. A summation of the work in transverse direction and a calculation the cumulative sum in flow direction delivers the evolution of the flow field-related work done along the droplet.

$\mathcal{W}_{u}^{*}=\sum_{V} \frac{\left(V \cdot \eta \boldsymbol{\nabla}^{2} \mathbf{u}\right) \cdot \mathbf{u} \Delta t}{\left(\Delta p_{\mathrm{HP}, \mathrm{c}} Q_{\mathrm{tot}}\right) \Delta t}$

\subsection{Momentum thickness}

We divide the pressure field into a wall-proximate part and a core-flow to support the description of the Taylor flow phenomenology. The simple flow field of a laminar single-phase flow through a straight pipe solely evolves due to the wall contact of the fluid. In contrast, the flow in and around a Taylor droplet is more complex due to the additional presence of the droplet interfaces.

Thus, we calculate the momentum thickness $\delta_{2}$ of a reference Poiseuille flow in a circular microchannel (Fig. 4) to geometrically discriminate between an immediate wall influenced region and the remaining core of the flow. Details on the momentum thickness are provided by (Schlichting and Gersten 2016).

The calculation of momentum thickness $\delta_{2}$ returns a defined length scale at a fixed geometric fraction of the channel height

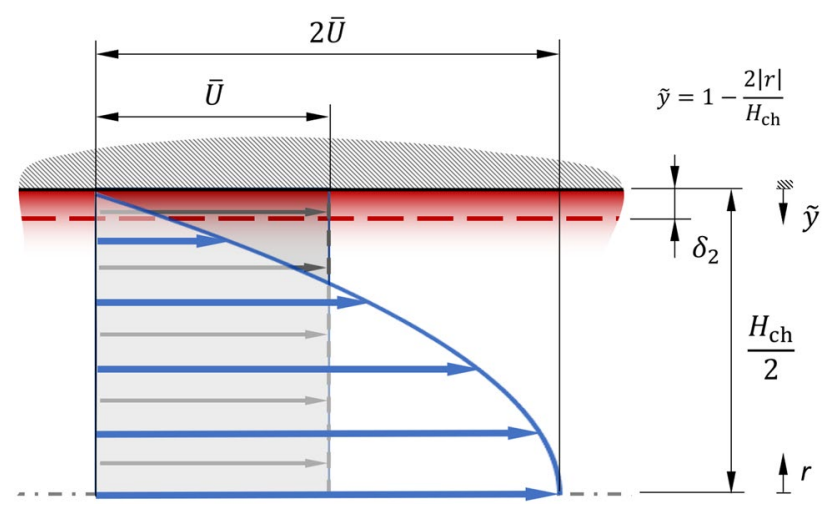

Fig. 4 Sketch of the flow conditions in laminar pipe flows. The momentum thickness $\delta_{2}$ quantifies a distance in which the flow is dominated by the presence of the wall. The thickness enables to geometrically discriminate between wall-influenced flow regions and the core of the flow. The layer thickness amounts to $\delta_{2}=\frac{1}{15} H_{\mathrm{ch}}$ ) $\delta_{2}=\frac{1}{15} H_{\mathrm{ch}}$

Three cases are used to set the momentum thickness $\delta_{2}$ into perspective: The normalized analytical $2 \mathrm{D}$ velocity profile of the single-phase Poiseuille flow through the cross-section of a circular microchannel (Fig. 5a), through a square microchannel (Fig. 5b) and a measured stream-wise velocity profile at the central cross-section of a Taylor droplet's main vortex (Fig. 5c). For the latter case, half of the microchannel is depicted, since the measurement data cover only half of the flow volume.

For better comparability, the 2D shear distributions are normalized with the wall shear stress of a laminar flow in a circular microchannel $\tau_{\text {ref }}=\frac{8 \bar{U}}{H_{c h}}$. Values close to one show regions where the shear profile of the respective case equals the reference value (Fig. 5d-e).

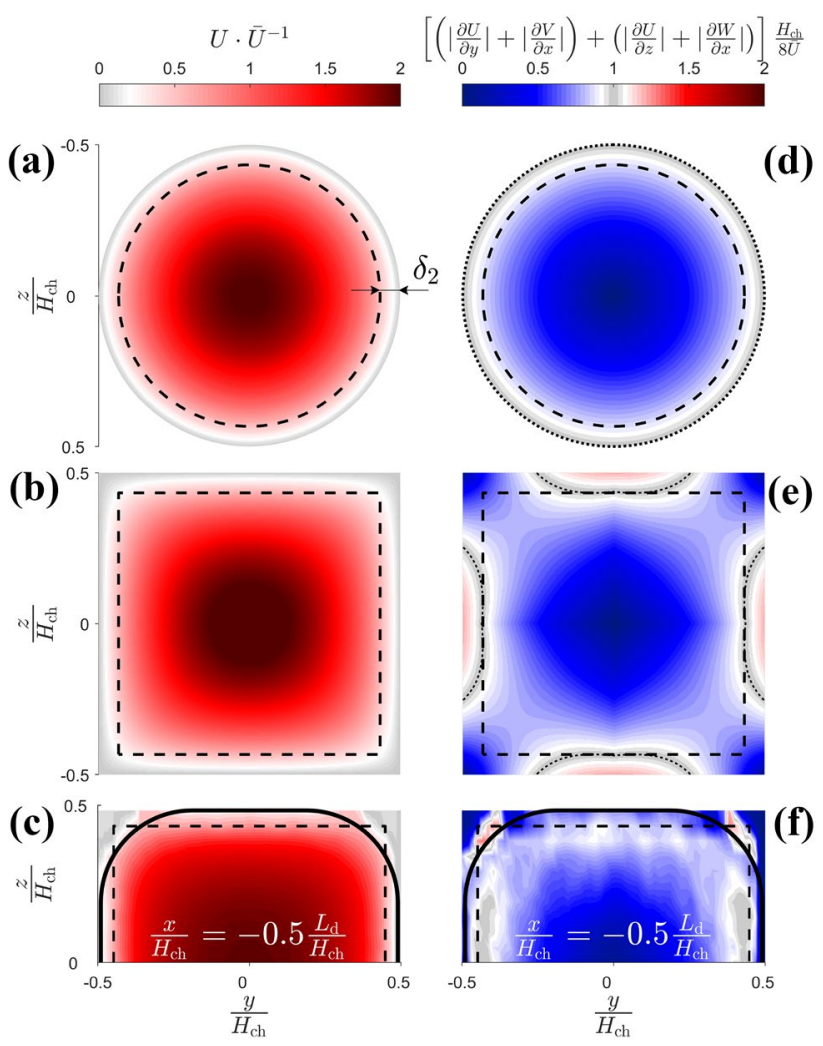

Fig. 5 Cross-sectional comparison between the analytical solutions of laminar single-phase flows in circular (a,d) and square channels (b, e) with measurements of a Taylor flow in a square channel in one half of the symmetric channel $(\mathbf{c}, \mathbf{f})$. The cross-section of the droplet flow is placed at the center of the main vortex in the droplet, where the velocities are mainly directed in flow direction (see streamlines Fig. 8a). The interface is marked with a solid black line. The velocity distribution is shown on the left (a-c), and the shear rate distribution is given on the right $(\mathbf{d}-\mathbf{f})$. The dashed black line indicates the momentum thickness $\delta_{2}$. The shear rates in the square channel for single-phase flows (e) and for the two-phase flow (f) equal the wall-shear rate of the pipe flow at a distance of $\approx \delta_{2}$ on the $y$ - and z-axis 
A dashed line represents the border of the wall-influenced flow layer. As expected, the reference shear and the wallshear agree at the wall in the circular channel (Fig. 5d, dotted line). The normalized shear distribution in the laminar single-phase flow of a square channel shows increased shear at the center of the side walls compared to the circular channel (Fig. 5e, dotted line). The equality of the shear profile to the reference-shear moves inwards to the position of the momentum thickness $\delta_{2}$. The same observation holds true for the measured case of the Taylor droplet (Fig. 5f). The solid black line indicates the interface position. In the gutter, additional shear is present outside the droplet due to the by-pass flow and the high viscosity ratio between droplet and bulk $(\lambda=2.625)$. The magnitude of the shear distribution is not ideally symmetric in the y-direction at the top wall, because the reconstruction of the velocity z-component is subject to integration error and integration of noise error. A comparison of the well-resolved measurement in the $\mathrm{y}$-direction with the reconstructed results in the z-direction shows a similar trend of an increased shear in proximity of the momentum thickness. Therefore, we consider the layer thickness $\delta_{2}$ to be a valid measure to discriminate between wall-dominated regions and the core of a Taylor flow.

\section{Results and discussion}

In this section, we provide the $3 \mathrm{D}$ pressure field and the overall energy loss of a Taylor droplet moving in a rectangular horizontal microchannel on the basis of an experimentally acquired 3D3C velocity field. While the pressure field is accessible through a direct integration of the simplified Navier-Stokes equation, the evaluation of the pressure loss from the pressure field data is reduced to the mean pressure profile at the wall along the droplet. For comparison two additional methods establish other measures for the pressure loss: Shear and drag forces at the wall and droplet interface as well as the work done on the flow to maintain stationarity against the viscous dissipation of energy.

In this context, a comparison between the pressure distribution and the curvature-derived Laplace-pressure on the interface of a Taylor droplet identifies the source of the motion-related droplet deformation. Next, the pressure gradient that drives the by-pass flow through the gutters is quantified, and an easy-to-access estimation method is verified. The analysis of the 3D pressure distribution inside and outside the Taylor droplet explains the mean pressure profile in stream-wise direction. Finally, we discuss the energy dissipation of the droplet based on the 3D distribution of the work done on the Taylor flow.

In the following sections, the Taylor droplets move in the positive $\mathrm{x}$-direction. Their front tip is situated in the origin of the coordinate system, while their back cap tip is located at $x / H_{\mathrm{ch}}=-1.58$.

\subsection{Pressure difference on the droplet interface}

The flow field in and around a moving Taylor droplet deforms the interface from its shape at rest. Abiev (2017) suggests to make use of the flow related interface deformation to estimate the pressure distribution. We use the geometry-based model data and a flow-based approach based on experimental data to quantify and compare the pressure difference on the droplet interface in Fig. 6. The pressure differences are normalized with the driving pressure for a single-phase Poiseuille-flow of continuous phase material $\left(Q_{\mathrm{HP}}, c=Q_{\mathrm{tot}}\right)$ through an equivalent channel cross section of droplet length $L_{\mathrm{d}}$. A positive pressure refers to forces that act on a area element $\mathrm{d} A$ against the outwards pointing surface normals (Fig. 3b): As a result, the pressure pushes the interface inwards. The interface is moved outwards at regions of negative pressure. The green arcs mark the geometric entrances and exits of the gutters.

Figure 6a shows the Laplace-pressure difference (Eq. 14) derived from the geometric interface approximation. The interface approximation produces artifacts when used as the source for second-order information (e.g. the interface curvature). The 2D Laplace-pressure profile on the interface exhibits discontinuities at the joints of different interface parts: (i) at the entrances and exits of the gutter and the wall-film and (ii) at the joints of the wall-films. In addition, the droplet interface at the wall-film does not show any sign of deformation which is in contradiction to the findings of Kreutzer et al. (2018). Both, the artifacts and the absence of pressure in wall-films are the result of the simplification of the geometric boundary conditions and the assumptions that allowed to retrieve geometric first-order information, like the location of the Taylor droplet interface.

Despite these drawbacks, qualitative information on the droplet deformation from its static shape can be observed. The elongation of the front cap is caused by a suction region at the droplet's tip (blue) and followed by a region of positive pressure that moves the interface inwards. At the droplet's back cap the interface is initially pulled outwards behind the gutter exits and wall-film regions and subsequently compressed at the tip. Both qualitative results correspond to the cap deformation described by the experimentally derived correlation of Helmers et al. (2019a).

Figure $6 \mathrm{~b}$ depicts the pressure difference derived from the velocity field (Eq. 5 used in Eq. 3) at the interface of the Taylor droplet. In addition to the pressure information, the streamlines of the interface motion are shown (Mießner et al. 2020).

The white area at the top wall-film artificially closes the droplet. The interface gap exists due to a lack of 


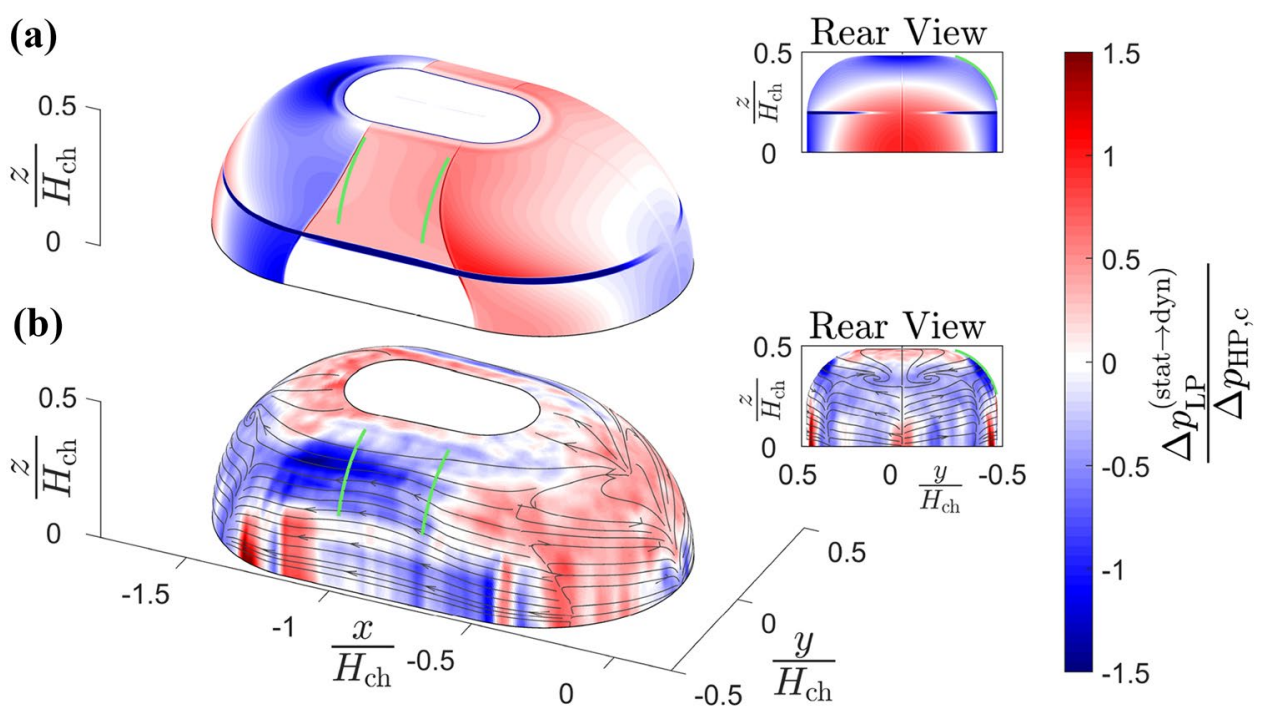

Fig. 6 Pressure distribution on the interface of a moving Taylor droplet. The approximate interface shape model of Mießner et al. (2019) delivers the position of the dynamically deformed interface. Positive pressure (red) pushes the interface inwards (Fig. 3b). The interface is moved outwards at regions of negative pressure (blue). The green lines indicate the entrance and exit of the gutter that accommodates the bypass flow of the continuous phase. A rear view of the respective pressure distribution on the droplet interface enhances comparability. a The difference between the curvature distribution of a Taylor

measurement data. However, the flow symmetry allows access to the pressure conditions in the films at the side wall-film instead.

The effect of the front cap elongation and the back cap compression is clearly present in the measurement-based pressure. The general magnitude range of the pressure distribution is comparable between the two approaches. The cap deformations arise from the relative motion of the main vortices in the slugs. The ring shape stagnation region at the droplet front indicates the location where the slug's walldriven vortex attaches to the interface, while it detaches again at the droplet front tip. The pressure rises due to the viscous displacement that takes place between attachment and detachment. The influence of the detachment is smaller than predicted by the geometric representation (Fig. 6a). At the droplet back, inverted flow conditions with respect to the interface cause the back cap compression.

The pressure evolution from the wall-film entrance at the droplet front towards its rear exit shows a series of pressure changes. At the frontal stagnation region, the liquid of the continuous phase is either redirected to the droplet tip or in the direction of the wall-film. At the onset of the film the outer liquid is forced into the film and the pressure rises. After the transport of fluid through the film, the filmthinning increases the pressure. At the exit of the film, the liquid flows towards the ring-shaped stagnation region at the back, where it slows down again. This series of pressure droplet at rest and in steady motion allows to calculate the pressure distribution from the Laplace-pressure of both cases. The result relies entirely on the accuracy of the curvature of the interface approximation. b The 3D velocity data of the $\mu$ PIV measurement of Mießner et al. (2020) is used in the Navier-Stokes equations to calculate the pressure distribution on the interface of a moving droplet. Additionally, the fluid motion on the $2 \mathrm{D}$ interface is interpolated and represented with streamlines

changes feed back to the interface and coincide with the location where the onset of the bullet-shape for increased $\mathrm{Ca}_{c}$ is situated (Taha and Cui 2006).

The pressure distribution along the gutter appears to be different compared to the geometric approach. While the magnitudes of the pressure at the gutter entrances are similar, the distribution in gutters deviates. The geometric approach delivers positive Laplace-pressures, while the flow-based pressure carries a negative sign. However, in both cases the pressure decreases from the front to the back of the gutter. Thus, the pressure gradient points into the same direction-against the flow direction of the Taylor droplets. This confirms the postulated pressure gradient inversion with respect to the flow direction by Abiev (2017).

\subsection{Pressure gradient of the by-pass flow in the gutter}

An estimation of the pressure gradient along the gutter allows the determination of the volume flow through the gutter and calculation of the relative velocity (Helmers et al. 2019b). Based on the droplet interface geometry, two possibilities to analytically obtain the pressure along the gutter are feasible: an evaluation of (i) the curvature difference distribution between the static and the dynamic Taylor droplet shape (Fig. 6a, Eq. 14) and (ii) the Laplace-pressure difference between the mean gutter radius at the front and back of 
the gutter. The gutter radii are calculated as a by-product of the interface approximation (Mießner et al. 2019).

The measurement-based pressure difference is used as a reference to asses the accuracy of the theoretical approaches (Fig. 7). For this purpose, the distribution in the gutter is averaged in transverse direction (blue triangles). The measurement resolution in the $\mathrm{x}$-direction of the source velocity field serves as sampling grid distance in flow direction. The schematic inset of Fig. 7 visualizes the sampling method for the gutter gradient. The offset of pressure at the back end of the gutter is removed. The result is normalized with the driving pressure of a single-phase Poiseuille-flow of continuous phase material $\left(Q_{\mathrm{HP}}, c=Q_{\mathrm{tot}}\right)$ through an equivalent channel of gutter length $L_{\mathrm{g}}$ to quantify the influence of the droplets presence.

A linear fit to the averaged pressure difference (blue solid line) allows to estimate the overall gutter pressure gradient of the experiment. The confidence interval of the slope $( \pm 9.1 \%)$ is calculated to quantify the influence of the standard deviation of the measurement from the linear fit on the gradient (blue area).

The Laplace-pressure difference due to the geometric droplet deformation is averaged in the same manner (o), and a linear fit allows to estimate the pressure gradient in the gutter. Clearly, the slope of the model surface fit is half as steep as the pressure gradient of the measurement is $(-55.4 \%)$. We attribute the deviation between Laplace pressure difference of the entrances gutter (black dashed line) and the model's mean curvature distribution (black dotted line) to the simplifications introduced to calculate the grid points of the interface shape. Thus, the curvature difference of the approximate interface cannot be used to estimate the gutter gradient.

In contrast, the calculation of the gutter gradient with the Laplace-pressure difference between the mean gutter radius at the front and back of the gutter leads to a suitable estimation (Fig. 7, dashed line). The result lies well inside the variance of the measured slope $(+2.7 \%)$. The gray area indicates the sensitivity to a $\pm 25 \%$ slope change of the gutter radius approach. Thus, the approach of Helmers et al. (2019b) to estimate the driving pressure gradient of the gutter from the gutter radii is confirmed by the results presented here.

\subsection{D pressure field}

In disregard of a reference pressure and of the Laplace-pressure at rest, the presented 3D field solely shows the pressure that drives the flow inside and outside of the Taylor droplet. The flow is directed from left to right. Streamlines in a frame of reference relative to the droplet motion are given on half
Fig. 7 Mean pressure gradient that drives the bypass flow through the gutter as a function of gutter lengths. Offsets are removed to directly compare between measurement data (blue) and two model-based estimations of the pressure gradient in the gutter. The linear fit of the measurement data (solid blue line) is used as reference. The blue area visualizes the uncertainty of the slope $( \pm 9.1 \%)$, based on the deviation of the linear fit (blue line) from the measurement (blue triangles). The gutter radii at the entrance and exit of the gutter (green lines on the interface) are used to directly estimate the pressure gradient. The slope (black dashed line) agrees with the measured pressure gradient along the gutter. The gray area visualizes the sensitivity of the pressure gradient on the gutter radius estimation $( \pm 25 \%)$

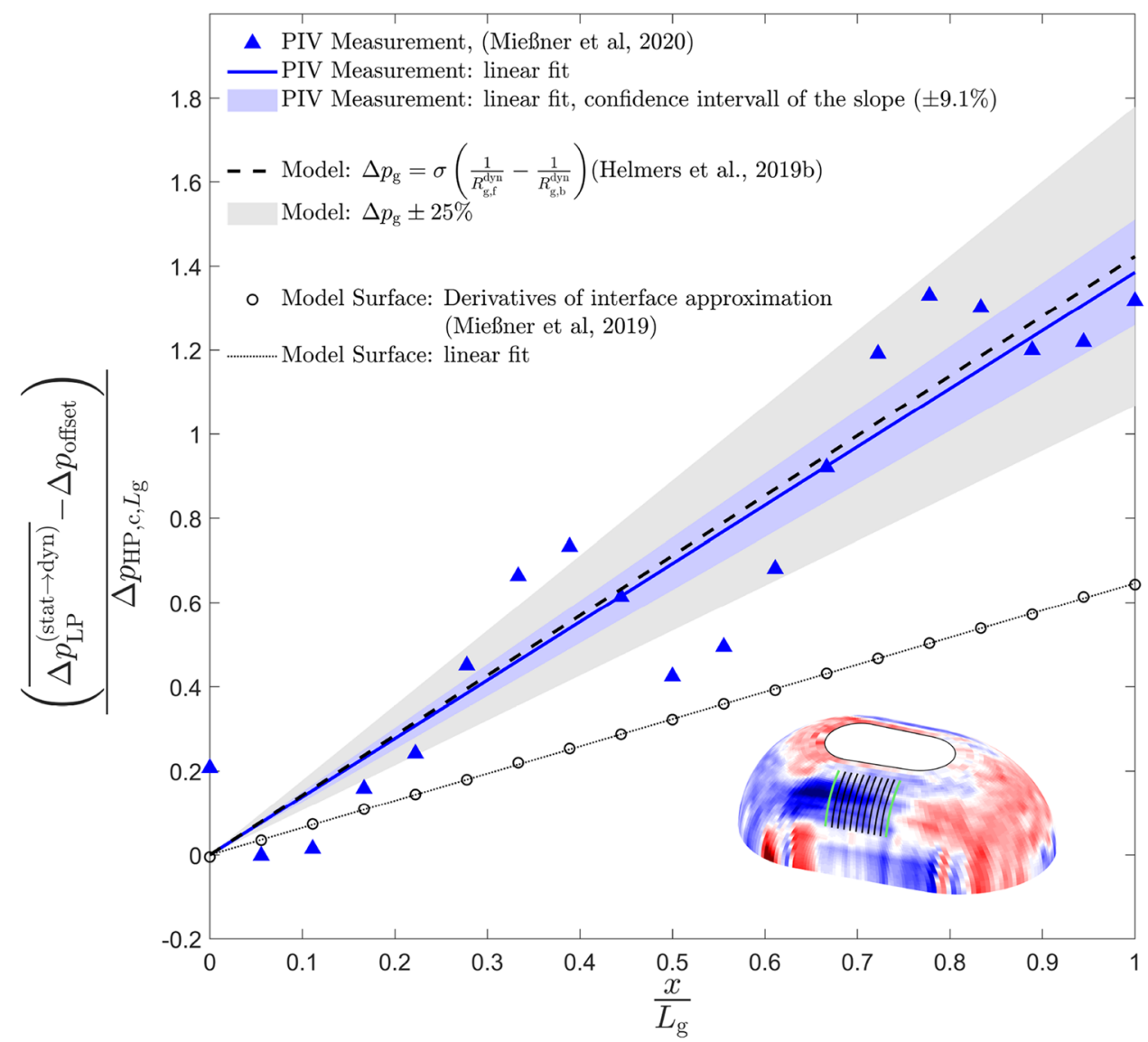


of the central symmetry plane (Fig. 8a). Bernoulli's principle does not apply, since the viscous forces are 20 times stronger than the inertia forces $\left(\operatorname{Re}_{c}=0.05\right.$, Stokes-flow conditions).

The pressure field of the Taylor droplet generally shows two different regions for its evolution: The pressure field in a layer close to the wall $\delta_{2}$ and in the remaining core of the field. Inside the wall-proximate layer, the pressure decreases as expected in stream-wise direction. However, the direction of the pressure gradient reverses in the core of the flow.

In the core flow, the pressure builds up at the droplet front due to viscous displacement. The outer main vortex in the slug is forced to change direction due to the interface at the droplet front. This elevated pressure in front of the droplet is the actual pressure source that drives the by-pass flow through the gutters (Abiev 2017) and gives rise to the relative velocity.
The wall-driven main vortex B inside the droplet strongly changes the flow direction to evade the secondary vortices $(\mathrm{A}, \mathrm{C})$ that connect the inner to the outer field. Two regions of the droplet's main vortex inner region B stand out: the converging rear develops a positive pressure, while the diverging front shows a negative sign.

The fundamental change of pressure conditions between the outer layer $\delta_{2}$ and the core of the flow is attributed to the presence of shear at the wall (Fig. 8a). In the case of the experimental conditions applied here, the thickness of the wall-proximate layer amounts to $\delta_{2}$. Perpendicular to the center plane (Fig. 8a), four sections are chosen to investigate the pressure evolution from the wall (Fig. 8b) towards the core of the flow (Fig. 8e). The black line indicates the position of the Taylor droplet interface. The pressure distribution at the wall (Fig. 8b) correlates with the droplet motion: increased pressure on the left causes the droplet motion
Fig. 8 Pressure in- and outside a moving Taylor droplet. The distribution on the $\mathrm{x} / \mathrm{y}$-symmetry plane (a) shows an increased pressure in front of the droplet due to viscous displacement in comparison with the droplet rear. This pressure difference drives the flow through the gutter. The curved thick black line shows the position of the interface. The ring-vortices are labeled $\mathrm{A}$, $\mathrm{B}$ and $\mathrm{C}$ from the droplet front to the back, with B denoting the main wall-driven vortex and $\mathrm{A}$ and $\mathrm{C}$ indicating the secondary vortices in the respective droplet caps. The thick dashed line delimits the wall-proximate momentum-layer at $\delta_{2}$. The thin black lines indicate the location of perpendicular pressure field sections (b-e) inside the wall-layer. The diagrams on the right $(\mathbf{f}-\mathbf{i})$ show the mean pressure along the $\mathrm{x}$-axis of the according pressure field section. The linear fits (solid red lines) show a change of pressure gradient (slope) towards an inverted pressure gradient in the core flow as a function of the wall distance. The inversion of the gradient is reached at a wall distance of about $\tilde{y}=\frac{\delta_{2}}{H_{\mathrm{ch}}}$

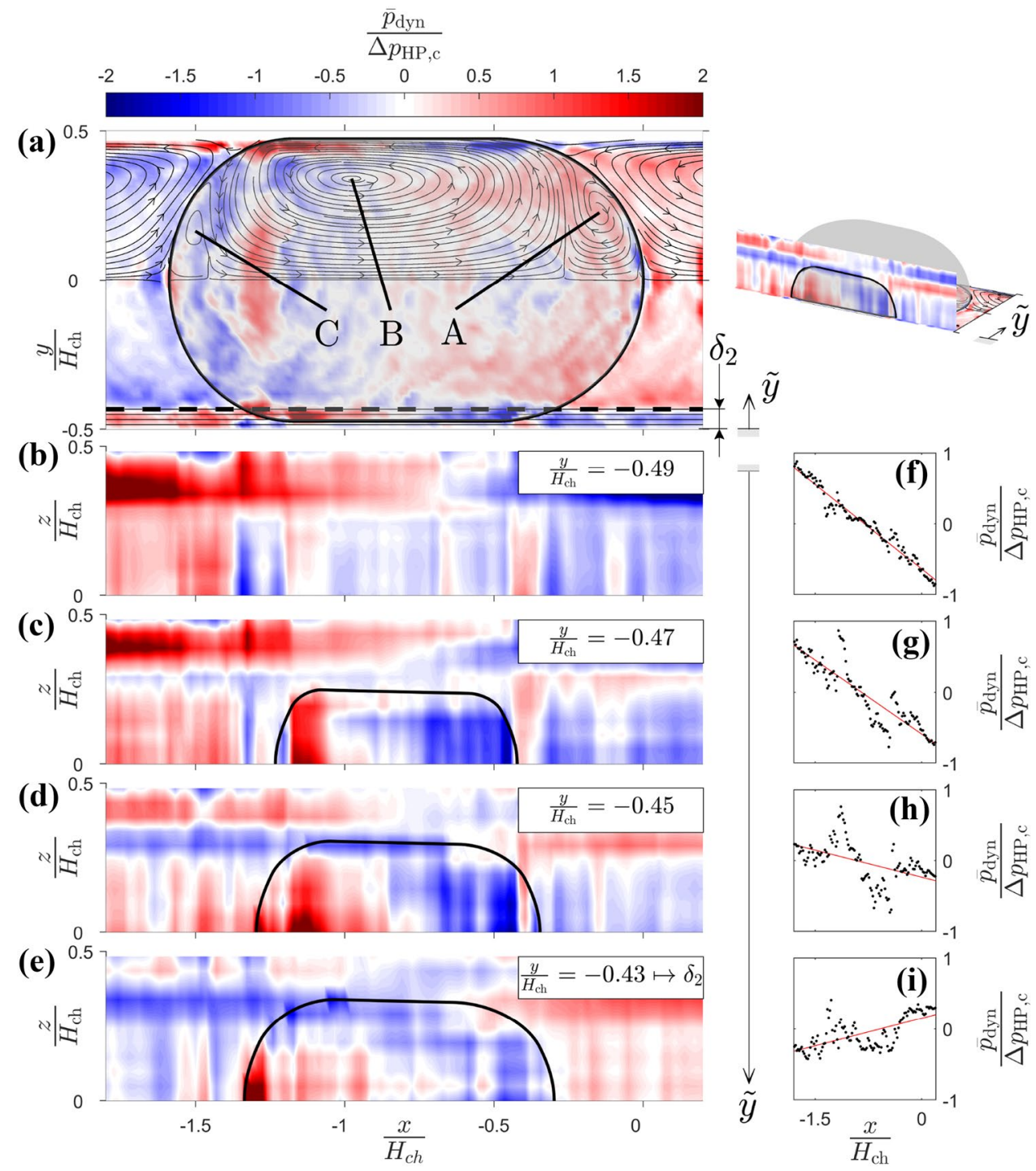


downstream towards the right, while showing a decreasing pressure level.

To emphasize the change of the pressure gradient from the wall towards the core of the flow (Fig. 8b-e), the pressure is averaged in the $\mathrm{z}$-direction to receive a pressure profile in downstream direction (Fig. 8f-i). A linear fit illustrates the change of pressure gradient (red lines). An inversed pressure gradient with respect to the flow direction is present at a wall distance of $\tilde{y}=\frac{\delta_{2}}{H_{\mathrm{ch}}}=1 / 15$. It seems that the core flow is moved through the microchannel and causes the shear-related pressure loss in the wall-layer. Inside the core flow, the viscous displacement of the recirculating main ring-vortices give rise to an elevated pressure.

In Fig. 9, the consideration of mean pressure profiles along the downstream direction provides an overview for the discussion of the pressure. For this purpose, we discriminate between the wall-proximate layer of thickness $\delta_{2}$ and the core of the flow as well as between droplet and bulk. The color-coding indicates the disperse phase with red and the continuous phase with blue, while the core of the flow is represented with a thick line and the wall-layer with a thin

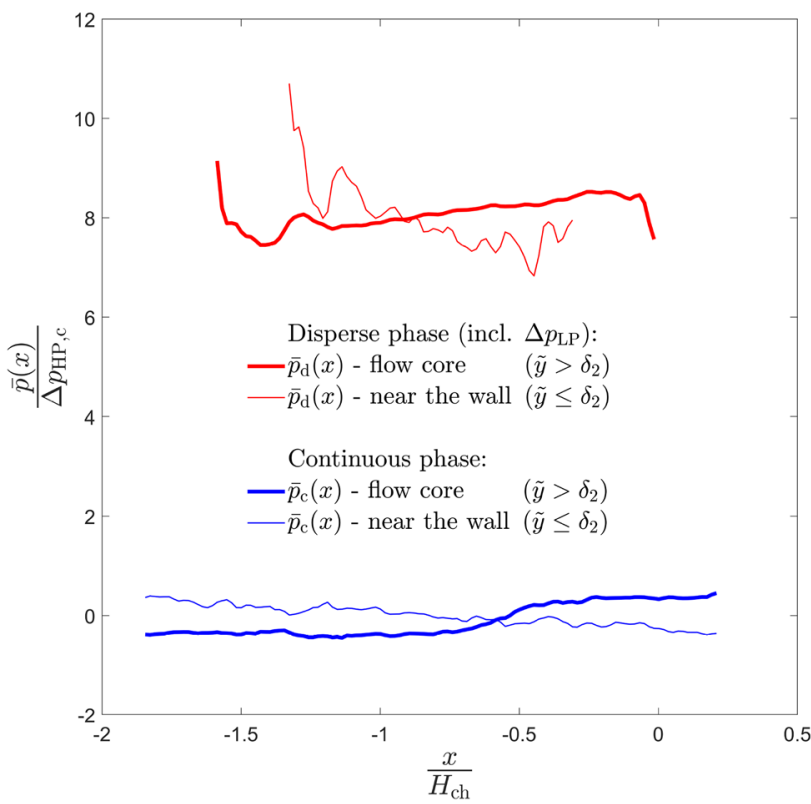

Fig. 9 Mean pressure profile evolution along x-axis of a moving Taylor droplet including the Laplace-pressure jump. The pressure inside (red lines) and outside (blue lines) of the droplet is divided into wallshear dominated region (thin lines) and the core of the two-phase flow (thick lines). The flow is oriented in the positive $\mathrm{x}$-direction. Inside a wall distance of $\tilde{y} \leq \delta_{2}$, an expected pressure gradient can be observed: the pressure decreases from the droplet back to its front (thin lines). However, viscous displacement in the core of the flow (thick lines) causes an inversion of the pressure gradient from front to back (Abiev 2017). The inversion drives the bypass flow of the continuous phase from the droplet front to the back and is the cause of the relative velocity of the Taylor droplet (Helmers et al. 2019b) line. The constant contribution of the Laplace-pressure is added to the droplet's pressure profiles to visualize the pressure jump at the interface.

Inside the wall-layer of both phases (Fig. 9, thin lines) the mean pressure decreases as expected in downstream direction. The elevated droplet viscosity $\lambda=\eta_{\mathrm{d}} / \eta_{\mathrm{c}}=2.625$ causes a higher pressure drop in the momentum layer of the droplet in comparison with the layer of the continuous phase.

In the core flow of the droplet, a clear reversed pressure gradient is present in either phase (Fig. 9, thick lines). An expected maximum of the pressure is found at the rear stagnation point of the droplet (red thick line). The pressure fluctuation induced by the rear secondary vortex is followed by an almost linear pressure increase. The pressure fluctuations of the frontal secondary ring-vortex end in a local minimum at the frontal singularity of the droplet.

The evolution of the mean pressure in the core flow of the continuous phase (Fig. 9 thick blue line) along the droplet also shows the reversed pressure gradient. Most of the profile is almost constant from the back to the front of the droplet. The reversed pressure increase that drives gutter flow is located between the frontal gutter entrance and the frontal onset of the wall-film. Further downstream, the pressure is almost constant again. This again confirms the postulated pressure gradient inversion with respect to the flow direction by Abiev (2017).

\subsection{D distribution of work done on the flow}

The distribution of the work done on the flow allows to locate the major contributions to the energy loss of the flow. For visualization reasons, the distribution of the work done $\mathcal{W}^{*}$ is scaled with the number of sampling points $\Pi_{i} N_{i}$ of the velocity field (Fig. 10). The droplet moves in the positive $\mathrm{x}$-direction.

The work distribution in the central symmetry plane is shown in Fig. 10a. The interface shape is indicated with a thick black line. The streamlines relate the flow field to the distribution of work done on the flow.

Deceleration work (blue) is mainly performed due to the directional change of downstream the velocity component of the flow field. Consequentially, the peak deceleration is located at the annular stagnation regions, where the fluid of the main vortices is forced to reverse direction.

Acceleration work is performed (red) in the caps of the Taylor droplet and inside the wall-influenced layer $\delta_{2}$. The counter rotating secondary vortices $(\mathrm{A}, \mathrm{C})$ in the caps are driven by and receive their energy from the adjoining wallinduced main vortices. Except for the strong directional change of the main vortices, the fluid close to the wall is accelerated due to the no-slip condition at the wall. The layer-thickness of apparent acceleration amounts roughly 
Fig. 10 Work done on the flow in- and outside a moving Taylor droplet. The color-coding uses red to show work done for acceleration and blue for the deceleration of fluid. The curved thick black line represents the droplet interface. The distribution of work in the flow field of a moving Taylor droplet on the $\mathrm{x} / \mathrm{y}$-symmetry plane (a) shows that the secondary vortices $(\mathrm{A}, \mathrm{C})$ inside the caps are mainly accelerated by the flow, while the center of the main vortex B is constantly decelerated. The fluid close to the wall is mainly accelerated since the wall-shear impact on the steadily moving Taylor droplet needs to be compensated for. The thick dashed line delimits the wall-proximate momentum layer $\delta_{2}$. The wall-shear-related acceleration is situated inside the wall layer $\delta_{2}$ at the wall $(\mathbf{b}-\mathbf{e})$. The thin black lines show the location of perpendicular work distribution sections near the wall $\left(\tilde{y} \leq \frac{\delta_{2}}{H_{0}}\right)$. The diagrams on the right $(\mathbf{f}-\mathbf{i})$ show the mean work distribution along the $\mathrm{x}$-axis of the according sections of the field. The mean work done to accelerate the fluid increases at first with growing wall distance and decreases again towards a wall distance of $\delta_{2}$

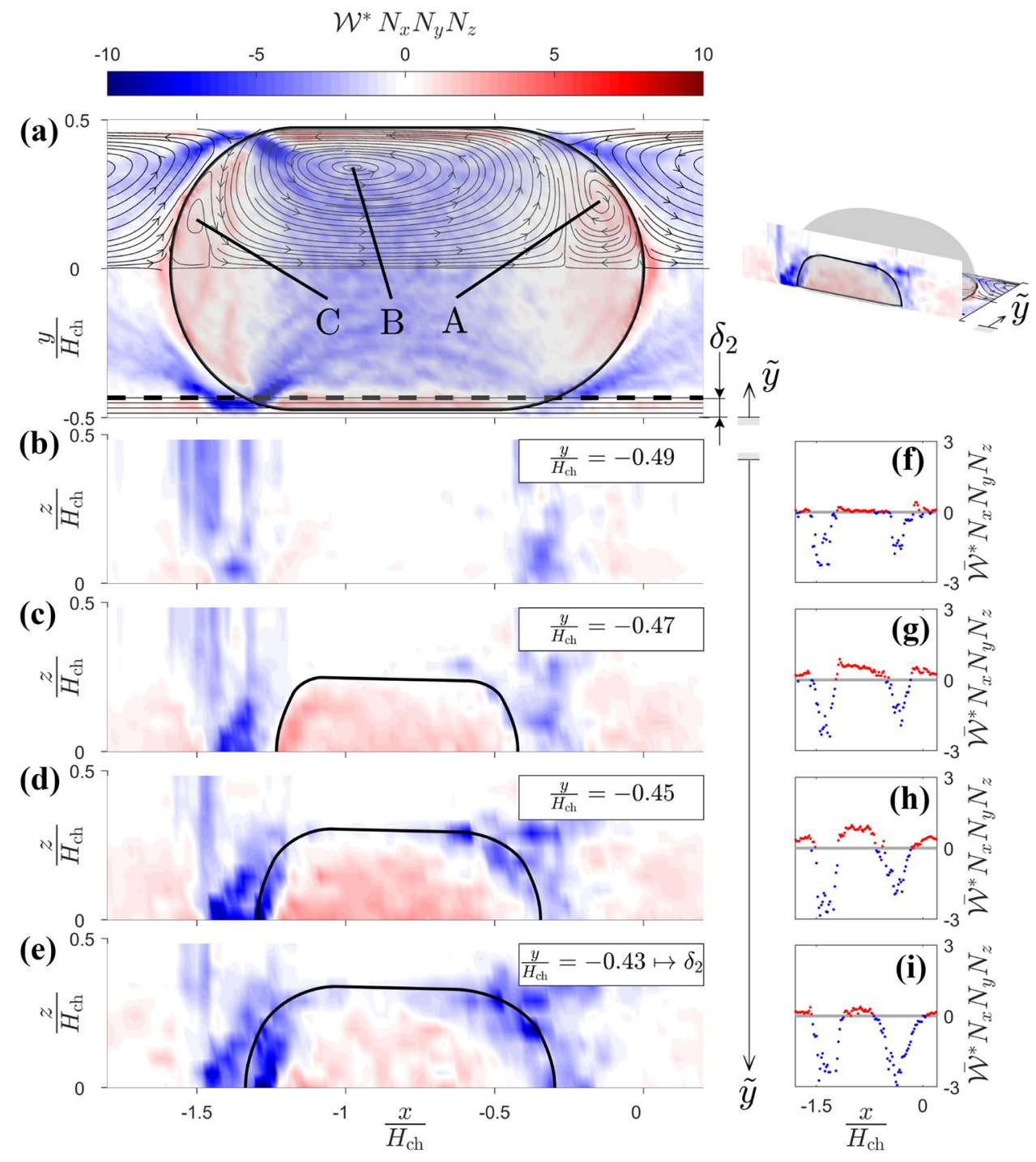

to $\delta_{2}$ and coincides with the momentum layer thickness where also the wall-related pressure gradient is situated (Fig. 8a).

Inside this layer, wall-proximate sections through the field of work are presented in Fig. 10b-e. It is noteworthy that in transverse direction the acceleration regions are spread mainly over the width of the wall-film of the Taylor droplet. With increasing wall distance $\tilde{y}$ the deceleration of the flow grows where the droplet interface redirects the flow.

The respective z-averaged profiles of work done on the flow $\mathcal{W}^{*}$ along the flow direction are given in Fig. 10f-i. Moving step-wise inwards from the wall, the acceleration intensity increases between $\mathrm{f}-\mathrm{g}$, and decreases when reaching $\delta_{2}$ (Fig. 10i). In the case of this experiment, we consider the momentum layer thickness $\delta_{2}$ to be a valid measure to distinguish the wall-proximate flow from the core of the Taylor flow.

\subsection{Energy dissipation of a Taylor droplet}

We show and discuss the cumulated energy dissipation of a Taylor droplet, which is in its dimensionless form equivalent to the overall pressure drop of the Taylor droplet (Eq. 12). Thus, we quantify the cumulated work done on the flow, because the energy loss of a Taylor flow due to viscous dissipation equals the work done on the flow to maintain the flow stationary.

Since we provide indirect and derived data on the loss of energy, we apply and compare the results of three different methods. These calculations are based on (i) the resistance forces at the interface of the droplet and the microchannel wall $\mathcal{W}_{F}^{*}$, (ii) the actual work done on the flow field $\mathcal{W}_{u}^{*}$ and (iii) the pressure loss at the channel wall $\mathcal{W}_{\Delta p \text {,wall }}^{*}$. As reference quantity, we use the work done on a comparable single-phase flow $\mathcal{W}_{\text {ref }}$ (Eq. 10). The discussion of the results always begins at the tip of the rear cap 
$\left(x / H_{\mathrm{ch}}=-L_{d} / H_{\mathrm{ch}}=-1.58\right)$ and proceeds in flow direction (positive $\mathrm{x}$-direction).

(i) The cumulated work that is necessary to overcome the resistance forces at the interfaces is given in Fig. 11. The thin gray and black lines represent the work done on the gutter and the wall-film of the Taylor droplet interface $\Gamma$ (Fig. 2), respectively. A summation of the latter results the red line, which shows the force-related total work done on the droplet interface displacement. Only those parts of the interface that are parallel to the flow direction contribute to the force-related flow resistance. The actual gutter and wall-film do not contribute. The forces at the front and back caps provide the main resistance, which agrees with the observations and findings of Bretherton (1961), who related the interface forces to the pressure drop. The blue line shows the contribution of the cumulated shear forces at the wall that needs to be overcome by the flow. The shear-related work done $\mathcal{W}_{F, \tau}^{*}$ shows an almost linear contribution to the resistance along the entire droplet.

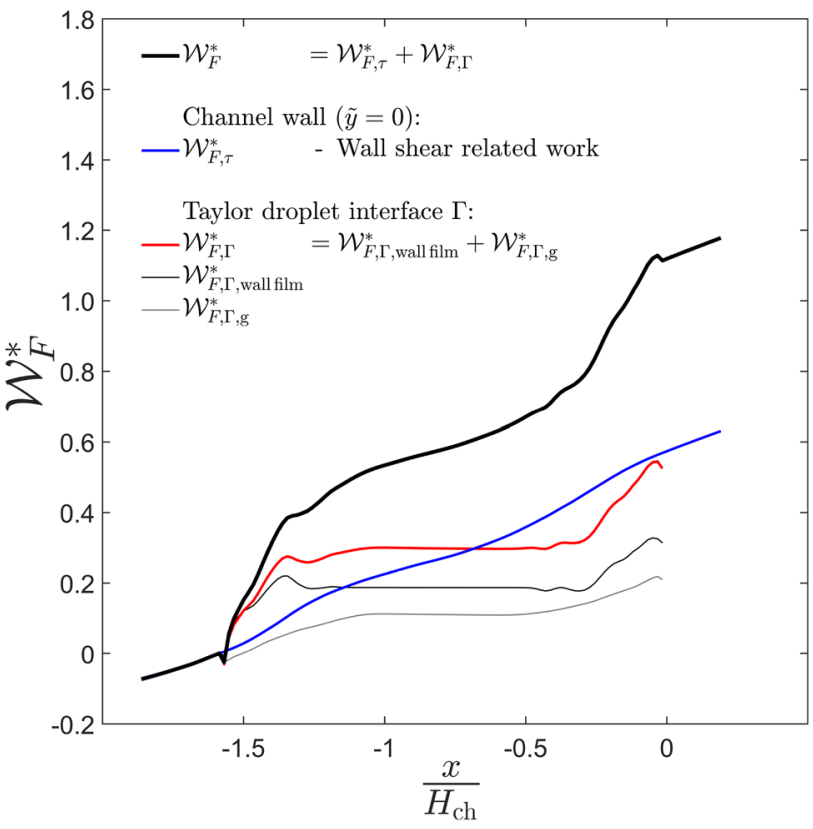

Fig. 11 Accumulated work due to drag forces from the onset of the rear cap $\left(x / H_{\mathrm{ch}}=-1.58\right)$ towards the droplet front $\left(x / H_{\mathrm{ch}}=0\right)$. The accumulated forces along the gutter regions (thin light gray line) contribute less drag than the film regions (gray line). The addition of the interface forces of the gutters to those of the films results the drag force evolution along the moving Taylor droplet (red line). The shear forces at the channel walls (blue line) also contribute to the energy loss of the Taylor flow. The addition of the interfacial forces and the wall-shear forces gives the overall drag force profile in flow direction (thick black line). In total, wall-shear forces and interface forces contribute almost evenly. While the shear forces exhibit a steady increase along the droplet, the contribution of the interface is mainly attributed to the cap regions
The influence of the entrances and exits of the wallfilms slightly raise the otherwise steady increase. The force contributions of the channel wall and the droplet interface show an almost equal magnitude at the front tip of the droplet. A combination of the influence of all force-related work results in the thick black line. At the tip of the front cap, the resistance forces require $\mathcal{W}_{F}^{*}\left(x / H_{\text {ch }}=0\right)=1.114$ times the work done on the reference single-phase flow.

(ii) Figure 12 shows the cumulated work done on the considered flow field (Eq. 17). The total work is given by the thick black line. The almost linear increase in the cumulated work along the Taylor droplet results in a magnitude of $\mathcal{W}_{u}^{*}\left(x / H_{\mathrm{ch}}=0\right)=1.458$ at the droplet tip. The contributions are presented differentiating between disperse phase and continuous phase (red and blue) as well as between the wall-layer $\delta_{2}$ and the core of the flow (thin and thick lines). The work done on the wallproximate layer of the disperse and continuous phase accounts for $2.7 \%$ and $5.9 \%$ of the total flow related work $\mathcal{W}_{u}^{*}$, respectively. Thus, the major contributor for the energy loss is the core of the flow with $91.4 \%$. At $\frac{x}{H_{\mathrm{ch}}}=0$, the dissipation contribution from the core of the disperse phase (thick blue line) amounts to $67.3 \%$

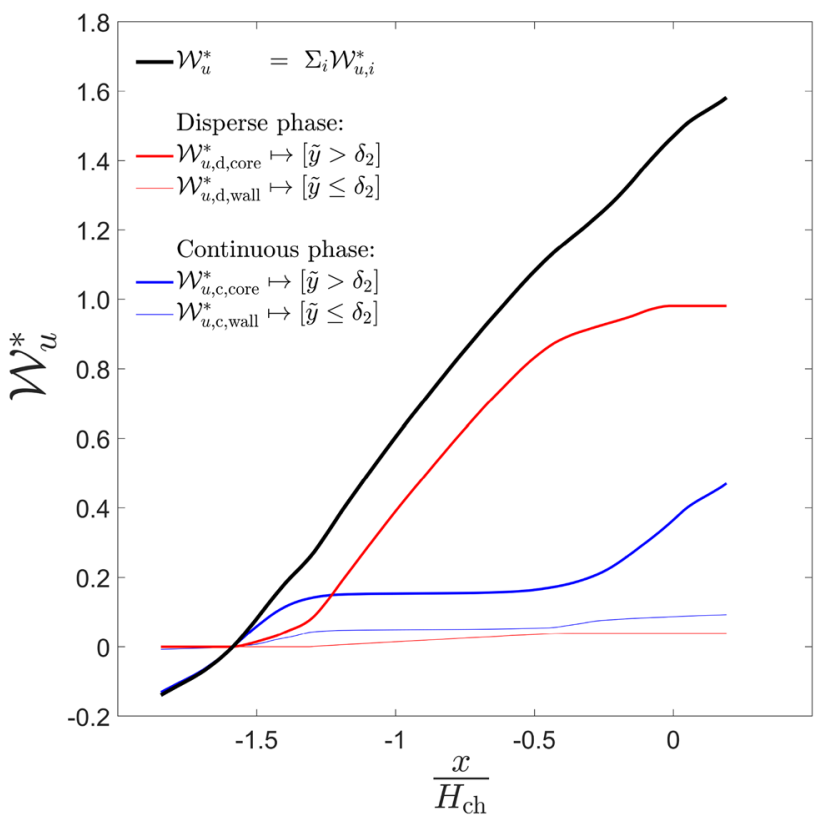

Fig. 12 Accumulated absolute work from the onset of the rear cap $\left(x / H_{\mathrm{ch}}=-1.58\right)$ towards the droplet front $\left(x / H_{\mathrm{ch}}=0\right)$. The flow is oriented in the positive $\mathrm{x}$-direction. The absolute work done inside (red lines) and outside (blue lines) the droplet is divided into wallshear dominated region (thin lines) and the core of the two-phase flow (thick lines). The sum of all contributions results the total work done (thick black line) that is necessary to sustain the stationary twophase flow 
of the total work done on the flow (black line). The according contribution from the core of the disperse phase amounts to $24.3 \%$ of the total work done on the flow. The ratio of 2.77 between the work done contributed from the core flows corresponds remarkably well to the viscosity ratio $\lambda=2.625$. The finding that the droplet contributes less with the cap recirculation, while the continuous phase adds the major amount of work at the caps, is mainly attributed to the changed cross-section area ratio in the droplet.

iii) A comparison between three approaches to calculate the total energy loss (Eq. 15-17) along the flow direction is presented in Fig. 13. The work received from the 3D flow field is given by the thick black line. The thick gray line indicates the shear and drag forces-based work at the channel walls and the droplet interface. Gray dots show a wall pressure-based approach to calculate the energy loss (Sec. 2.6b). As a first order simplification a linear fit (dashed line) allows to determine the total energy loss. The light gray area indicates $\pm 10 \%$ deviation from the linear fit. Judged from the total energy loss at the front tip of the Taylor droplet $\left(x / H_{\mathrm{ch}}=0\right)$, the drag and shear forces alone deliver the lowest estimate of the energy $\operatorname{loss} \mathcal{W}_{F}^{*}=1.11=0.76 \mathcal{W}_{u}^{*}$. The linearized wall-pressure

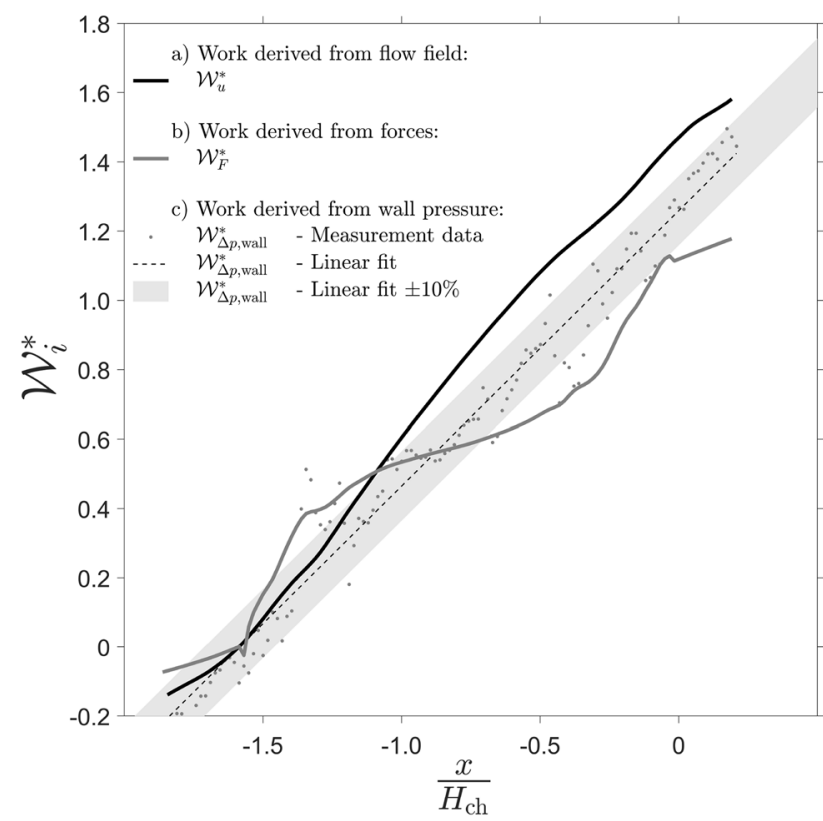

Fig. 13 Comparison between different dimensionless indicators of the work: The cumulated work derived from the flow field (a) is compared to the shear and drag forces at the channel walls and the droplet interface (b) and the work estimated from the pressure profile at the wall (c). All quantities are referenced with the work of a Hagen-Poiseuille flow in a square pipe of equal cross section $A_{\mathrm{ch}}$ and channel length $L_{\mathrm{ch}}=L_{\mathrm{d}}$. The reference channel is filled with the continuous phase of the Taylor flow and experiences the volume flow of $Q_{\text {ref }}=Q_{\text {tot }}=\left(Q_{\mathrm{d}}+Q_{\mathrm{c}}\right)$ of the Taylor flow based approach estimates $\mathcal{W}_{\Delta p \text {,wall }}^{*}=1.25=0.86 \mathcal{W}_{u}^{*}$ performs better. The cumulated work done on the flow $\mathcal{W}_{u}^{*}$ amounts to 1.471 times the work done on the reference case of the single-phase flow in a square channel.

All presented approaches to quantify the energy dissipation of the two-phase flow are derivations of high order. They indirectly depend on the reconstructed 3D flow field to a variable degree: While pressure-based work $\mathcal{W}_{\Delta p \text {,wall }}^{*}$ involves double differentiation and an integration, the interface force-related work $\mathcal{W}_{F}^{*}$ is subject to additional conversion and integration steps. Since the work done on the flow $\mathcal{W}_{u}^{*}$ includes only double derivatives, we consider the result to be more reliable. From this perspective, the tendency towards lower magnitudes of total work done on the Taylor flow correlates with a decreasing accuracy due to the indirectness of the quantification approaches. In addition, the force-based approach $\mathcal{W}_{F}^{*}$ does solely include forces in flow direction and omits contributions of the transverse flow.

\section{Conclusion}

For the first time, the pressure field of a moving Taylor droplet has been extracted based upon experimental work. Despite the approximate character of the interface representation (Mießner et al. 2019) and the mentionable noise level of the processed experimental data (Mießner et al. 2020), we established a coherent overall picture of the 3D pressure field in and around a moving Taylor droplet.

The distinction between a wall-proximate momentum layer of thickness $\delta_{2}$ and a core flow reveals a reversal of the pressure gradient from the wall towards the center of the flow. In the wall-proximate momentum layer, the pressure decreases in flow direction as one would expect from a pressure-driven laminar single-phase flow. However, the pressure gradient reverses when reaching the core of the flow. Here, the flow is governed by viscous displacement and builds up a pressure gradient from the droplet front to its back. Thus, we confirm the expectation of a reversed pressure gradient, as stated by Abiev (2017).

We experimentally verify a straightforward estimation method to quantify the reversed pressure gradient that drives the bypass flow of the continuous phase through the gutters and causes the relative velocity of Taylor droplets (Helmers et al. 2019b). The approach overestimates the measured driving pressure gradient by $2.7 \%$.

The curvature-based Laplace-pressure distribution on the droplet interface is deduced from the interface approximation (Mießner et al. 2019). It resembles the measurementbased pressure distribution on the droplet interface only qualitatively. Thus, the interface approximation does not 
allow a precise calculation of second-order information like the curvature distribution. However, it does deliver accurate first-order information, e.g. the location, the volume and the surface area of dynamically deformed Taylor droplet interface.

The 3D distribution of work done on the flow is divided into acceleration and deceleration. The fluid inside the wallproximate momentum layer at the wall-films and inside the droplet caps is mainly accelerated, while the remaining flow undergoes deceleration. The constant change for the circulating liquid between acceleration and deceleration causes additional energy loss in comparison with the laminar flow in a straight pipe.

As a measure for the overall energy loss of the moving droplet, the drag forces at the droplet and channel interface are compared to the cumulative work done on the flow field to overcome internal friction. The comparison shows that a consideration of the drag forces alone does not deliver all information on the losses of the flow. The governing viscous forces that act inside and outside the Taylor droplet also contribute to the overall energy loss. The energy loss calculated from the work done on the flow is $31.6 \%$ higher than the result received from the approach based on the drag-forces.

The 3D pressure field and the distribution of work done on the investigated Taylor flow enables benchmarking for numerical simulations and is published as supplementary material.

Supplementary Information The online version contains supplementary material available at https://doi.org/10.1007/s00348-021-03189-5.

Funding Open Access funding enabled and organized by Projekt DEAL.

Open Access This article is licensed under a Creative Commons Attribution 4.0 International License, which permits use, sharing, adaptation, distribution and reproduction in any medium or format, as long as you give appropriate credit to the original author(s) and the source, provide a link to the Creative Commons licence, and indicate if changes were made. The images or other third party material in this article are included in the article's Creative Commons licence, unless indicated otherwise in a credit line to the material. If material is not included in the article's Creative Commons licence and your intended use is not permitted by statutory regulation or exceeds the permitted use, you will need to obtain permission directly from the copyright holder. To view a copy of this licence, visit http://creativecommons.org/licenses/by/4.0/.

\section{References}

Abate AR, Mary P, van Steijn V, Weitz DA (2012) Experimental validation of plugging during drop formation in a t-junction. Lab Chip 12:1516-1521. https://doi.org/10.1039/C2LC21263C

Abiev RS (2011) Modeling of pressure losses for the slug flow of a gasliquid mixture in mini- and microchannels. Theor Found Chem Eng 45(2):156-163. https://doi.org/10.1134/S0040579511020011
Abiev R (2017) Analysis of local pressure gradient inversion and form of bubbles in taylor flow in microchannels. Chem Eng Sci 174:403-412. https://doi.org/10.1016/j.ces.2017.09.041

Antweiler N, Gatberg S, Jestel G, Franzke J, Agar DW (2016) Noninvasive sensor for the detection of process parameters for multiphase slug flows in microchannels. ACS Sens 1(9):1117-1123. https:// doi.org/10.1021/acssensors.6b00420

Balestra G, Zhu L, Gallaire F (2018) Viscous Taylor droplets in axisymmetric and planar tubes: from Bretherton's theory to empirical models. Microfluid Nanofluidics 22(6):355. https://doi. org/10.1007/s10404-018-2084-y

Bandara T, Nguyen NT, Rosengarten G (2015) Slug flow heat transfer without phase change in microchannels: a review. Chem Eng Sci 126:283-295. https://doi.org/10.1016/j.ces.2014.12.007

Baur T (1999) PIV with high temporal resolution for the determination of local pressure reductions from coherent turbulence phenomena. In: Proc. 3rd Int. Workshop on PIV - Santa Barbara, pp 101-106, https://ci.nii.ac.jp/naid/10011561312/en/

Bretherton FP (1961) The motion of long bubbles in tubes. J Fluid Mech 10(2):166-188. https://doi.org/10.1017/S002211206 1000160

Brücker C (1995) Digital-particle-image-velocimetry (DPIV) in a scanning light-sheet: $3 \mathrm{~d}$ starting flow around a short cylinder. Exp Fluids 19(4):255-263. https://doi.org/10.1007/BF00196474

Brücker C (1997) 3d scanning PIV applied to an air flow in a motored engine using digital high-speed video. Meas Sci Technol 8(12):1480-1492. https://doi.org/10.1088/0957-0233/8/12/011

Cai Z, Liu Y, Chen T, Liu T (2020) Variational method for determining pressure from velocity in two dimensions. Exp Fluids 61(5):118. https://doi.org/10.1007/s00348-020-02954-2

Charonko JJ, King CV, Smith BL, Vlachos PP (2010) Assessment of pressure field calculations from particle image velocimetry measurements. Meas Sci Technol 21(10):105401. https://doi. org/10.1088/0957-0233/21/10/105401

Chou WL, Lee PY, Yang CL, Huang WY, Lin YS (2015) Recent advances in applications of droplet microfluidics. Micromachines 6(9):1249-1271. https://doi.org/10.3390/mi6091249

Claxton D (2006) Surface curvature. https://www.mathworks.com/ matlabcentral/fileexchange/11168-surface-curvature

Garstecki P, J Fuerstman M, Fischbach MA, Sia SK, Whitesides GM (2006) Mixing with bubbles: a practical technology for use with portable microfluidic devices. Lab Chip 6:207-212. https://doi. org/10.1039/B510843H

Gurka R, Liberzon A, Hefetz D, Rubinstein D, Shavit U (1999) Computation of pressure distribution using PIV velocity data. In: Proc. 3rd Int. Workshop on PIV - Santa Barbara

Helmers T, Kemper P, Thöming J, Mießner U (2019a) Determining the flow-related cap deformation of Taylor droplets at low ca numbers using ensemble-averaged high-speed images. Exp Fluids 60(7):113. https://doi.org/10.1007/s00348-019-2757-7

Helmers T, Kemper P, Thöming J, Mießner U (2019b) Modeling the excess velocity of low-viscous Taylor droplets in square microchannels. Fluids. https://doi.org/10.3390/fluids4030162

Jakiela S (2016) Measurement of the hydrodynamic resistance of microdroplets. Lab chip 16(19):3695-3699. https://doi.org/10. 1039/c6lc00854b

Jaw SY, Chen JH, Wu PC (2009) Measurement of pressure distribution from PIV experiments. J Vis 12:27-35. https://doi.org/10. 1007/BF03181940

Jin Y, Orth A, Schonbrun E, Crozier KB (2012) Measuring the pressures across microfluidic droplets with an optical tweezer. Opt Express 20(22):24450-24464. https://doi.org/10.1364/OE.20. 024450

Jovanović J, Zhou W, Rebrov EV, Nijhuis TA, Hessel V, Schouten JC (2011) Liquid-liquid slug flow: hydrodynamics and pressure 
drop. Chem Eng Sci 66(1):42-54. https://doi.org/10.1016/j.ces. 2010.09.040

De Kat R, van Oudheusden BW (2012) Instantaneous planar pressure determination from PIV in turbulent flow. Exp Fluids 52(5):1089-1106. https://doi.org/10.1007/s00348-011-1237-5

Koschatzky V, Moore PD, Westerweel J, Scarano F, Boersma BJ (2011) High speed PIV applied to aerodynamic noise investigation. Exp Fluids 50(4):863-876. https://doi.org/10.1007/ s00348-010-0935-8

Kreutzer MT, Kapteijn F, Moulijn JA, Heiszwolf JJ (2005) Multiphase monolith reactors: chemical reaction engineering of segmented flow in microchannels. Chem Eng Sci 60(22):5895-5916. https:// doi.org/10.1016/j.ces.2005.03.022

Kreutzer MT, Kapteijn F, Moulijn JA, Kleijn CR, Heiszwolf JJ (2005b) Inertial and interfacial effects on pressure drop of taylor flow in capillaries. AIChE J 51(9):2428-2440. https://doi.org/10.1002/ aic. 10495

Kreutzer MT, Shah MS, Parthiban P, Khan SA (2018) Evolution of nonconformal Landau-Levich-Bretherton films of partially wetting liquids. Phys Rev Fluids 3(014):203. https://doi.org/10.1103/ PhysRevFluids.3.014203

Ładosz A, von Rohr PR (2018) Pressure drop of two-phase liquidliquid slug flow in square microchannels. Chem Eng Sci 191:398409. https://doi.org/10.1016/j.ces.2018.06.057

Lang P, Hill M, Krossing I, Woias P (2012) Multiphase minireactor system for direct fluorination of ethylene carbonate. Chem Eng J 179:330-337. https://doi.org/10.1016/j.cej.2011.11.015

Leung SS, Liu Y, Fletcher DF, Haynes BS (2010) Heat transfer in well-characterised Taylor flow. Chem Eng Sci 65(24):6379-6388. https://doi.org/10.1016/j.ces.2010.09.014

Liu X, Katz J (2006) Instantaneous pressure and material acceleration measurements using a four-exposure PIV system. Exp Fluids 41(2):227. https://doi.org/10.1007/s00348-006-0152-7

Lockhart RW, Martinelli RC (1949) Proposed correlation of data for isothermal 2-phase, 2-component flow in pipes. Chem Eng Prog 45(1):39-48

Mac Giolla Eain M, Egan V, Howard J, Walsh P, Walsh E, Punch J (2015) Review and extension of pressure drop models applied to Taylor flow regimes. Int J Multiph Flow 68:1-9. https://doi.org/ 10.1016/j.ijmultiphaseflow.2014.09.006

Mießner U, Helmers T, Lindken R, Westerweel J (2019) An analytical interface shape approximation of microscopic Taylor flows. Exp Fluids 60(4):75. https://doi.org/10.1007/s00348-019-2719-0

Mießner U, Helmers T, Lindken R, Westerweel J (2020) $\mu$ PIV measurement of the $3 \mathrm{~d}$ velocity distribution of Taylor droplets moving in a square horizontal channel. Exp Fluids 61(5):125. https://doi.org/ $10.1007 / \mathrm{s} 00348-020-02949-\mathrm{z}$

Molla S, Eskin D, Mostowfi F (2011) Pressure drop of slug flow in microchannels with increasing void fraction: experiment and modeling. Lab Chip 11(11):1968. https://doi.org/10.1039/c01c00478b

Nayak R, Lobo OJ, Chatterjee D, Das SK (2018) Effect of geometrical parameters on slug behaviour and two phase pressure drop in microchannel t-junctions. Chem Eng Process Process Intensif 130:76-87. https://doi.org/10.1016/j.cep.2018.05.017

Piao Y, Han DJ, Azad MR, Park M, Seo TS (2015) Enzyme incorporated microfluidic device for in-situ glucose detection in waterin-air microdroplets. Biosens Bioelectron 65:220-225. https://doi. org/10.1016/j.bios.2014.10.032
Romero PA, Abate AR (2012) Flow focusing geometry generates droplets through a plug and squeeze mechanism. Lab Chip 12(24):5130-5132. https://doi.org/10.1039/c2lc40938k

Schlichting H, Gersten K (2016) Boundary-layer theory, 10th edn. Springer, Berlin, Heidelberg. https://doi.org/10.1007/ 978-3-662-52919-5

Seemann R, Brinkmann M, Pfohl T, Herminghaus S (2011) Droplet based microfluidics. Rep Prog Phys 75(1):016601. https://doi.org/ 10.1088/0034-4885/75/1/016601

van Steijn V, Kleijn CR, Kreutzer MT (2009) Flows around confined bubbles and their importance in triggering pinch-off. Phys Rev Lett 103(214):501. https://doi.org/10.1103/PhysRevLett.103. 214501

Taha T, Cui Z (2006) Cfd modelling of slug flow inside square capillaries. Chem Eng Sci 61(2):665-675. https://doi.org/10.1016/j. ces.2005.07.023

Tronchin T, David L, Farcy A (2015) Loads and pressure evaluation of the flow around a flapping wing from instantaneous $3 \mathrm{~d}$ velocity measurements. Exp Fluids 56(1):7. https://doi.org/10.1007/ s00348-014-1870-x

Vivekanand SVB, Raju VRK (2019) Effect of wall contact angle and carrier phase velocity on the flow physics of gas-liquid taylor flows inside microchannels. Chem Pap 73(5):1173-1188. https:// doi.org/10.1007/s11696-018-0668-3

Wang X, Riaud A, Wang K, Luo G (2015) Pressure drop-based determination of dynamic interfacial tension of droplet generation process in t-junction microchannel. Microfluid Nanofluidics 18(3):503-512. https://doi.org/10.1007/s10404-014-1449-0

Wang X, Yong Y, Yang C, Mao ZS, Li D (2014) Investigation on pressure drop characteristic and mass transfer performance of gas-liquid flow in micro-channels. Microfluid Nanofluidics 16(12):413-423. https://doi.org/10.1007/s10404-013-1226-5

Ward T, Faivre M, Abkarian M, Stone HA (2005) Microfluidic flow focusing: drop size and scaling in pressure versus flow-rate-driven pumping. Electrophoresis 26(19):3716-3724. https://doi.org/10. 1002/elps.200500173

Whitesides GM (2006) The origins and the future of microfluidics. Nature 442:368-373. https://doi.org/10.1038/nature05058

Wolf A, Hartmann T, Bertolini M, Schemberg J, Grodrian A, Lemke K, Förster T, Kessler E, Hänschke F, Mertens F, Paus R, Lerchner J (2015) Toward high-throughput chip calorimetry by use of segmented-flow technology. Thermochim Acta 603:172-183.

Xu K, Tostado CP, Xu JH, Lu YC, Luo GS (2014) Direct measurement of the differential pressure during drop formation in a co-flow microfluidic device. Lab Chip 14(7):1357-1366. https://doi.org/ $10.1039 / \mathrm{c} 31 \mathrm{c} 51222 \mathrm{c}$

Yue J, Rebrov EV, Schouten JC (2014) Gas-liquid-liquid three-phase flow pattern and pressure drop in a microfluidic chip: similarities with gas-liquid/liquid-liquid flows. Lab Chip 14(9):1632-1649. https://doi.org/10.1039/c31c51307f

Publisher's Note Springer Nature remains neutral with regard to jurisdictional claims in published maps and institutional affiliations. 